Könözsy, L., Drikakis, D.

A Unified fractional-step, artificial compressibility and pressure-projection formulation for solving the incompressible Navier-stokes equations

(2014) Communications in Computational Physics, 16 (5), pp. 1135-1180.

Commun. Comput. Phys.

doi: $10.4208 /$ cicp.240713.080514a

Vol. $x$, No. x, pp. 1-46

$x x x 20 x x$

\title{
A Unified Fractional-Step, Artificial Compressibility and Pressure-Projection Formulation for Solving the Incompressible Navier-Stokes Equations
}

\author{
László Könözsy ${ }^{1, *}$ and Dimitris Drikakis ${ }^{1}$ \\ ${ }^{1}$ Fluid Mechanics and Computational Science, Cranfield University, Cranfield, \\ Bedfordshire, MK43 0AL, United Kingdom.
}

Received xxx; Accepted (in revised version) xxx

Available online $\mathrm{xxx}$

\begin{abstract}
This paper introduces a unified concept and algorithm for the fractionalstep (FS), artificial compressibility (AC) and pressure-projection (PP) methods for solving the incompressible Navier-Stokes equations. The proposed FSAC-PP approach falls into the group of pseudo-time splitting high-resolution methods incorporating the characteristics-based (CB) Godunov-type treatment of convective terms with PP methods. Due to the fact that the CB Godunov-type methods are applicable directly to the hyperbolic AC formulation and not to the elliptical FS-PP (split) methods, thus the straightforward coupling of CB Godunov-type schemes with PP methods is not possible. Therefore, the proposed FSAC-PP approach unifies the fully-explicit AC and semi-implicit FS-PP methods of Chorin including a PP step in the dual-time stepping procedure to a) overcome the numerical stiffness of the classical AC approach at (very) low and moderate Reynolds numbers, $b$ ) incorporate the accuracy and convergence properties of CB Godunov-type schemes with PP methods, and c) further improve the stability and efficiency of the AC method for steady and unsteady flow problems. The FSAC-PP method has also been coupled with a non-linear, full-multigrid and fullapproximation storage (FMG-FAS) technique to further increase the efficiency of the solution. For validating the proposed FSAC-PP method, computational examples are presented for benchmark problems. The overall results show that the unified FSAC-PP approach is an efficient algorithm for solving incompressible flow problems.
\end{abstract}

AMS subject classifications: 76D05, 65M08, 65B99, 65Y20

Key words: Navier-Stokes equations, characteristics-based Godunov-type scheme, unified method.

*Corresponding author. Email addresses: laszlo.konozsy@cranfield.ac.uk (L. Könözsy),

d.drikakis@cranfield.ac.uk (D. Drikakis)

http:/ /www.global-sci.com/

1

(C)20xx Global-Science Press

Published by Global Science Press. This is the Author Accepted M anuscript.

This article may be used for personal use only. The final published version is available online at 10.4208/cicp.240713.080514a. Please refer to any applicable publisher terms of use. 


\section{Introduction}

This paper introduces a unified solution concept of the fractional-step (FS), artificial compressibility (AC) and pressure-projection (PP) methods for solving the incompressible Navier-Stokes equations. The development of the unified FSAC-PP method [1] is carried out within the framework of Godunov-type methods [1,2]. Due to the fact that the characteristics-based (CB) Godunov-type methods are applicable directly to the hyperbolic system of the AC formulation and not to the elliptical FS-PP (split) methods, thus the straightforward coupling of CB Godunov-type schemes with PP methods is not possible. To take advantage of the accuracy and convergence properties of CB Godunov-type schemes, and increase the stability and efficiency of the classical AC method at (very) low and moderate Reynolds numbers, the FSAC-PP approach is proposed.

The AC method was developed by Chorin [3] introducing a perturbed continuity equation based on a pseudo-time derivative for the pressure. This equation has no physical meaning until the steady-state solution is achieved [4]. The AC method has good numerical features for stationary flows, but for unsteady and/or low Reynolds number flows, the stability condition of the dual-time stepping procedure and the choice of the AC parameter can become too restrictive, thus leading to slow convergence rates [4].

For solving unsteady, incompressible flows, Chorin [5] and Temam [6] introduced the FS-PP method based on the orthogonality theorem of Ladyzhenskaya [7], which is also known as the Helmholtz-Hodge or Hodge decomposition [3,5, 8-11]. According to this theorem, a vector field can be decomposed into a solenoidal (divergence-free) part and an irrotational part. The first FS estimates an intermediate velocity field neglecting the pressure gradient term from the momentum equation. The second FS projects the predicted intermediate velocity field into a divergence-free (exact projection), or numerically nearly divergence-free (approximate projection) vector field relying on the solution of a pressure-Poisson equation. Kim and Moin [12] proposed an application of the FS-PP method to the solution of the three-dimensional, time-dependent incompressible NavierStokes equations. Perot [13] accomplished a detailed analysis on the FS-PP method to overcome the first-order temporal accuracy of the flow field solution by using a generalized block LU decomposition of the governing equations for the primitive variables.

In the last 20 years, researchers have also made efforts to combine various highresolution schemes with FS-PP methods for single- and variable-density flows $[8,14]$. Bell et al. [8] developed a second-order, exact projection method in conjunction with Godunov-type methods [15] for the unsteady, incompressible Navier-Stokes equations. For variable-density incompressible flows, Bell and Marcus [9] established a secondorder projection method, and Almgren et al. [10] introduced a conservative and adaptive projection method. Eberle [16] developed a CB scheme method for the three-dimensional compressible Euler equations, and the scheme was further extended by Drikakis et al. [17] for solving the incompressible Navier-Stokes equations. This scheme was also extended to three-dimensional incompressible flows $[18,19]$ in conjunction with a nonlinear, FMG-FAS algorithm, as well as to variable density flows [20-22]. Recently, Za- 
mzamian and Razavi [23] further investigated the numerical behaviour of a multidimensional, characteristics-based (MCB) upwind scheme on different benchmarks. In this paper, a CB Godunov-type scheme is employed for the discretization of the convective terms by using interpolation formulae from first- up to ninth-order at the cell interfaces [24-26]. The employed CB Godunov-type scheme is derived as being singledirectional in space $[17,20]$, and the arrangement of primitive variables is considered to be collocated on the structured computational mesh.

Within the framework of Godunov-type methods for incompressible flows, the intercell $\mathrm{CB}$ velocity components of the non-linear convective terms and the intercell $\mathrm{CB}$ pressure can be derived from the hyperbolic system of the $\mathrm{AC}$ formulation by applying the Riemann method [1,18-20]. The proposed FSAC-PP approach solves the perturbed continuity equation explicitly to estimate an initial pressure field for a CB Godunovtype treatment of the convective terms. Furthermore, the explicit solution of the perturbed continuity equation provides initial values to the implicit solution of the PP step in the dual-time stepping procedure. In the next pseudo-time splitting step, the proposed FSAC-PP approach estimates an intermediate velocity field by employing a CB Godunov-type scheme dropping out the pressure gradient term from the scalar momentum equations. Since the intercell pressure difference in the CB Godunov-type velocity components can be determined by the explicit solution of the perturbed continuity equation, therefore there is no theoretical barrier to drop out the pressure gradient term from the scalar momentum equations, as in the classical FS-PP method of Chorin [5] and Temam [6]. As a consequence, instead of the CB Godunov-type reconstruction of the pressure gradient term, the pressure field is approximated by solving a pressure-Poisson equation implicitly. In other words, the proposed FSAC-PP approach retains the inviscid CB Godunov-type counterpart of the AC method for the velocity components, then the velocity field is updated along the characteristics via the solution of a pressure-Poisson equation in each pseudo-time step. In this way, the FSAC-PP method unifies the dualtime stepping procedure of the AC formulation with the FS-PP (split) method in order to accelerate the satisfaction of the incompressibility constraint for the explicitly solved perturbed continuity equation. It is observed that the inclusion of the implicit PP step in the fully-explicit dual-time stepping procedure of the AC method can improve the convergence properties of the FSAC-PP algorithm, compared to the classical AC method.

Although, to our knowledge, there is no previous publication dealing with the proposed FSAC-PP formulation in conjunction with a CB Godunov-type method for incompressible constant density flows, it should be mentioned that Tang and Sotiropoulos [27] proposed a time-accurate approach on non-staggered grids using a second-order accurate, hybrid FSAC method to advance the efficiency of the original AC method. Their algorithm does not fall into the class of high-resolution Godunov-type methods, and in their case, the projection of the velocity field was carried out via a dual-time stepping of the AC formulation instead of solving a pressure-Poisson equation. Within the framework of finite element (FE) methods, there are important contributions by Zienkiewicz and his co-researchers [28-31] introducing the characteristic-based split (CBS) algorithm 
which relies on the characteristic-Galerkin method as a unified approach for compressible and incompressible flows. The fully explicit version of their procedure employs the features of the AC and FS (split) methods using explicit formulations to compute the pressure field and update the velocity components along the characteristics. The semi-implicit version of the CBS algorithm [32] is the application of Chorin's [5] and Temam's [6] FS-PP split concept with the implicit solution of a pressure-Poisson equation in the context of FE methods. Nithiarasu $[33,34]$ developed a fully-explicit CB split FE method, known as the CBS-AC scheme, which takes advantage of both standard AC and velocity correction approaches. Nithiarasu et al. [35-38] further extended the fullyexplicit and semi-implicit versions of the CBS scheme to different applications as a unified approach to fluid dynamics problems [36]. In the present work, the proposed FSACPP unified solution concept for incompressible flows is different from the previous works. The unification of Chorin's AC and FS-PP methods [3,5] and the solution algorithm are carried out differently compared to the other approaches discussed above. Furthermore, the finite volume (FV) CB Godunov-type method requires a different mathematical formulation and numerical treatment than the FE characteristic-Galerkin procedure.

The objectives of this paper are to: a) provide a numerical framework for accurate and efficient simulations at (very) low and moderate Reynolds number flows; $b$ ) overcome the numerical stiffness of the classical AC approach in certain flow regimes; c) incorporate the accuracy and convergence properties of CB Godunov-type schemes with PP methods; and d) further improve the efficiency of the standard AC method. Numerical results have been presented from the validation of the FSAC-PP method for benchmark problems. The computational examples have been discussed for a pressure-driven laminar flow in a straight microfluidic channel, and a two-dimensional stationary laminar and threedimensional unsteady turbulent flow problem in a lid driven cavity.

\section{A unified FSAC-PP method for incompressible flows}

In this Section, we develop a numerical procedure unifying the classical AC and FS-PP methods of Chorin $[3,5]$ in conjunction with a CB Godunov-type discretization scheme [17] for solving stationary and unsteady incompressible flow problems [1,2].

The three-dimensional, unsteady, incompressible Navier-Stokes equations by using conservative form of the convective term, are written with vector notation as

$$
\begin{aligned}
& \nabla \cdot \mathbf{u}=0, \\
& \frac{\partial \mathbf{u}}{\partial t}+\nabla \cdot(\mathbf{u} \otimes \mathbf{u})=\mathbf{g}-\frac{1}{\rho} \nabla p+v \nabla^{2} \mathbf{u},
\end{aligned}
$$

where $t$ is the physical time, $\mathbf{u}$ is the velocity field, $\mathbf{g}$ is the gravity field, $p$ is the hydrodynamic pressure, $\rho$ is the fluid density, and $v$ is the kinematic viscosity of the fluid. To non-dimensionalize the equations, the following dimensionless quantities are introduced for time, spatial coordinates, velocity components, and pressure field, where $L, u_{\infty}, \rho_{\infty}$ 
are constant reference quantities for characteristic length, velocity component and fluid density, respectively, and $R e$ is the Reynolds number as

$$
t^{*}=\frac{t u_{\infty}}{L}, X=\frac{x}{L}, Y=\frac{y}{L}, Z=\frac{z}{L}, U=\frac{u}{u_{\infty}}, V=\frac{v}{u_{\infty}}, W=\frac{w}{u_{\infty}}, P=\frac{p}{\rho_{\infty} u_{\infty}^{2}}, \quad R e=\frac{L U}{v} .
$$

By using these quantities and neglecting any external force field, the non-dimensionalized governing equations in a conservative form with vector notation are written as

$$
\begin{aligned}
& \nabla \cdot \mathbf{U}=0, \\
& \frac{\partial \mathbf{U}}{\partial t^{*}}+\nabla \cdot(\mathbf{U} \otimes \mathbf{U})=-\nabla P+\frac{1}{R e} \nabla^{2} \mathbf{U} .
\end{aligned}
$$

The numerical solution of the system of equations (2.3)-(2.4) is a difficult task in terms of the satisfaction of the divergence-free (incompressibility) constraint (2.3); furthermore an additional equation for the pressure does not exist. There are two classical solution ways for this system: 1) a hyperbolic-type standard AC method proposed by Chorin [3], and 2) an elliptical-type FS-PP (split) method introduced by Chorin [5] and Temam [6].

\subsection{Artificial compressibility (AC) method with characteristics-based (CB) Godunov-type treatment of the inviscid terms}

For stationary incompressible flows, Chorin [3] introduced a perturbed continuity equation, which contains a pseudo-time derivative for the pressure that has no physical meaning until the steady-state solution is achieved [4]. This method can also be used for unsteady flow problems by introducing pseudo-time derivatives for the velocity components in the scalar Navier-Stokes equations [20]. For unsteady incompressible flows using the dual-time stepping procedure, the AC formulation of the dimensionless hyperbolictype perturbed system of governing equations can be written in a vector form as

$$
\begin{aligned}
& \frac{1}{\beta} \frac{\partial P}{\partial \tau}=-\nabla \cdot \mathbf{U}, \\
& \frac{\partial \mathbf{U}}{\partial \tau}=-\frac{\partial \mathbf{U}}{\partial t^{*}}-\nabla \cdot(\tilde{\mathbf{U}} \otimes \tilde{\mathbf{U}})+\frac{1}{R e} \nabla^{2} \mathbf{U}-\nabla \tilde{P},
\end{aligned}
$$

where $\tilde{\mathbf{U}}$ and $\tilde{P}$ indicate the CB Godunov-type treatment of the inviscid terms, $\beta$ is the AC parameter relating to the numerical convergence, and $\tau$ is the locally computed pseudotime step. The characteristic velocity components and pressure are given $[17,20]$ by

$$
\tilde{\mathbf{U}}=\left[\begin{array}{c}
\tilde{U} \\
\tilde{V} \\
\tilde{W}
\end{array}\right]=\left[\begin{array}{c}
U_{0}+\frac{\tilde{X}}{2 s} R \\
V_{0}+\frac{\tilde{Y}}{2 s} R \\
W_{0}+\frac{\tilde{Z}}{2 s} R
\end{array}\right],
$$




$$
\begin{aligned}
& \tilde{P}=\frac{1}{2 s}\left\{\lambda_{1} P_{2}-\lambda_{2} P_{1}-\beta\left[\tilde{X}\left(\tilde{U}-U_{1}\right)+\tilde{Y}\left(\tilde{V}-V_{1}\right)+\tilde{Z}\left(\tilde{W}-W_{1}\right)\right]\right. \\
& \left.+\beta\left[\tilde{X}\left(\tilde{U}-U_{2}\right)+\tilde{Y}\left(\tilde{V}-V_{2}\right)+\tilde{Z}\left(\tilde{W}-W_{2}\right)\right]\right\},
\end{aligned}
$$

where sub-indexes " 1 " and " 2 " relate to the "Left" and "Right" hand side reconstructed intercell velocity and pressure values by using a high-order polynomial interpolation. To write the characteristic velocity components of the transport CB Godunov-type scheme (2.7) in a short form, an auxiliary function is introduced as

$$
\begin{aligned}
R=P_{1} & -P_{2}+\lambda_{2}\left[\tilde{X}\left(U_{0}-U_{1}\right)+\tilde{Y}\left(V_{0}-V_{1}\right)+\tilde{Z}\left(W_{0}-W_{1}\right)\right] \\
& -\lambda_{1}\left[\tilde{X}\left(U_{0}-U_{2}\right)+\tilde{Y}\left(V_{0}-V_{2}\right)+\tilde{Z}\left(W_{0}-W_{2}\right)\right] .
\end{aligned}
$$

By applying the Riemann method [39] for constant density flows [17,19,20], the coefficient matrix of the scalar system of governing equations (2.5)-(2.6) has distinct eigenvalues $\lambda_{0}=\tilde{U} \tilde{X}+\tilde{V} \tilde{Y}+\tilde{W} \tilde{Z}, \lambda_{1}=\lambda_{0}+s$ and $\lambda_{2}=\lambda_{0}-s$, where $s=\left(\lambda_{0}^{2}+\beta\right)^{1 / 2}$ is the artificial speed of sound, and $\tilde{X}, \tilde{Y}, \tilde{Z}$ are metric coefficients $[17,20]$. For low-speed incompressible flows, the AC parameter may be defined [2] as

$$
\beta \gg\left(1+\frac{4 L}{R e}\right)^{2}-1 .
$$

For computing the convective flux term in the momentum equation (2.6) relying on Eq. (2.7), the Godunov-type discretization is employed to compute the velocity components $U_{0}, V_{0}, W_{0}$ corresponding to the eigenvalue $\lambda_{0}$ as

$$
\mathbf{U}_{0}=\left[\begin{array}{c}
U_{0} \\
V_{0} \\
W_{0}
\end{array}\right]=\frac{1}{2}\left[\begin{array}{c}
U_{1}+U_{2}+\operatorname{sign}\left(\lambda_{0}\right)\left(U_{1}-U_{2}\right) \\
V_{1}+V_{2}+\operatorname{sign}\left(\lambda_{0}\right)\left(V_{1}-V_{2}\right) \\
W_{1}+W_{2}+\operatorname{sign}\left(\lambda_{0}\right)\left(W_{1}-W_{2}\right)
\end{array}\right],
$$

where

$$
\operatorname{sign}\left(\lambda_{0}\right)= \begin{cases}-1, & \lambda_{0}>0 \\ 1, & \lambda_{0}<0\end{cases}
$$

The Godunov-procedure consists of three main steps [2]: 1) high-order interpolation to approximate the "Left" and "Right" values of characteristic variables at the cell interfaces, 2) solution of the local Riemann-problem to obtain physically reasonable inviscid fluxes, and 3) characteristic flux differentiation to compute cell-averaged values. It is important to indicate, when the classical AC method is used in conjunction with a CB Godunov-type treatment of the inviscid terms, that Eq. (2.8) can be employed to compute the pressure gradient terms in the Navier-Stokes equations. Unlike when the subsequently proposed FSAC-PP approach is used, the cell-averaged pressure values are obtained by solving a pressure-Poisson equation in each pseudo-time step. 


\subsection{Formulation of the fractional-step (FS) pressure-projection (PP) method}

For unsteady incompressible flows, Chorin [5] and Temam [6] introduced an FS-PP method based on the Helmholtz-Hodge or Hodge decomposition [5-11]. Relying on this decomposition, a pressure-Poisson equation can be constructed $[5,6]$ by considering the pressure as a potential function of the irrotational velocity field. For numerical solutions, it is assumed that the correct pressure field is unknown in the scalar Navier-Stokes equations at time level $n$, thus the continuity equation (2.3) is not satisfied as

$$
\nabla \cdot \mathbf{U}^{(n)} \neq 0,
$$

but it is assumed that the continuity equation (2.3) is satisfied at time level $n+1$ as

$$
\nabla \cdot \mathbf{U}^{(n+1)}=0 .
$$

In the first FS, an intermediate velocity field $\hat{\mathbf{U}}$ is estimated dropping out the pressure gradient term from the scalar Navier-Stokes equations. The dimensionless momentum equation (2.4) via time-splitting can be written in a semi-discrete vector form as

$$
\frac{\hat{\mathbf{U}}-\mathbf{U}^{(n)}}{\Delta t^{*}}=-\nabla \cdot(\mathbf{U} \otimes \mathbf{U})^{(n)}+\frac{1}{R e} \nabla^{2} \mathbf{U}^{(n)},
$$

thus the intermediate velocity field is expressed by

$$
\hat{\mathbf{U}}=\mathbf{U}^{(n)}+\Delta t^{*}\left[-\nabla \cdot(\mathbf{U} \otimes \mathbf{U})+\frac{1}{R e} \nabla^{2} \mathbf{U}\right]^{(n)} .
$$

In the second FS, the pressure field is computed by taking into account the divergencefree constraint (2.14), thus the divergence of the

$$
\frac{\mathbf{U}^{(n+1)}-\hat{\mathbf{U}}}{\Delta t^{*}}=-\nabla P^{(n+1)}
$$

semi-discrete equation is a pressure-Poisson equation at time level $n+1[5,13]$ as

$$
\nabla^{2} P^{(n+1)}=\frac{1}{\Delta t^{*}} \nabla \cdot \hat{\mathbf{U}}
$$

To update the velocity field based on Eq. (2.17) satisfying the continuity equation (2.14), the second FS projects the intermediate velocity field (2.16) into a divergence-free (exactprojection) or numerically nearly divergence-free (approximate-projection) vector field via the solution of the pressure-Poisson equation (2.18) as

$$
\mathbf{U}^{(n+1)}=\hat{\mathbf{U}}-\Delta t^{*} \nabla P^{(n+1)} .
$$




\subsection{A unified FSAC-PP approach within the framework of high-resolution characteristics-based (CB) Godunov-type methods}

In this subsection, for stationary and unsteady incompressible flows, the proposed FSACPP approach [1] unifies the fully-explicit AC and semi-implicit FS-PP methods of Chorin $[3,5]$ within the framework of high-resolution CB Godunov-type methods [1,2]. This unified approach falls into the group of pseudo-time splitting high-resolution methods incorporating the CB Godunov-type treatment of convective terms with PP methods.

To unify the fully-explicit AC formulation with the semi-implicit FS-PP method, we drop out the $\mathrm{CB}$ pressure gradient term from the governing equations (2.5)-(2.6). Thus the dimensionless, dual-time stepping, perturbed system of the governing equations without the pressure gradient term can be written in a semi-discrete vector form as

$$
\begin{aligned}
& \frac{P^{(n)}-P^{(n-1)}}{\Delta \tau}=-\beta(\nabla \cdot \mathbf{U})^{(n)}, \\
& \frac{\hat{\mathbf{U}}-\mathbf{U}^{(n)}}{\Delta \tau}=-\frac{\partial \mathbf{U}^{(n)}}{\partial t^{*}}-\nabla \cdot(\tilde{\mathbf{U}} \otimes \tilde{\mathbf{U}})^{(n)}+\frac{1}{R e} \nabla^{2} \mathbf{U}^{(n)},
\end{aligned}
$$

where $\hat{\mathbf{U}}=\left[\begin{array}{lll}\hat{U} & \hat{V} & \hat{W}\end{array}\right]^{T}$ is the vector of an intermediate velocity field, and $\tilde{\mathbf{U}}$ is the $\mathrm{CB}$ Godunov-type velocity flux vector re-writing Eq. (2.7) in a form as

$$
\tilde{\mathbf{U}}^{(n)}=\mathbf{U}_{0}^{(n)}+\frac{\tilde{\mathbf{X}}}{2 s}\left(P_{1}-P_{2}+R^{*}\right)^{(n)},
$$

where the $\mathbf{U}_{0}^{(n)}$ vector components corresponding to the eigenvalue $\lambda_{0}$ are computed based on Eqs. (2.11) and (2.12), $\tilde{\mathbf{X}}=\left[\begin{array}{lll}\tilde{X} & \tilde{Y} & \tilde{Z}\end{array}\right]^{T}$ is the spatial vector of metrics, and

$$
\begin{aligned}
& R^{*}=\lambda_{2}\left[\tilde{X}\left(U_{0}-U_{1}\right)+\tilde{Y}\left(V_{0}-V_{1}\right)+\tilde{Z}\left(W_{0}-W_{1}\right)\right] \\
&-\lambda_{1}\left[\tilde{X}\left(U_{0}-U_{2}\right)+\tilde{Y}\left(V_{0}-V_{2}\right)+\tilde{Z}\left(W_{0}-W_{2}\right)\right]^{(n)}
\end{aligned}
$$

is an auxiliary function to write Eq. (2.22) in a short form. Since the transport CB Godunovtype scheme (2.22) is derived from the hyperbolic system of AC formulation (2.5)-(2.6) involving the pressure gradient term, therefore we can see in Eq. (2.22) that the intercell pressure values $P_{1}\left(\right.$ "Left") and $P_{2}$ ("Right") will appear in the convective terms $\nabla \cdot(\tilde{\mathbf{U}} \otimes \tilde{\mathbf{U}})^{(n)}$. As the intercell pressure difference $\left(P_{1}-P_{2}\right)^{(n)}$ of the CB Godunov-type flux vector (2.22) can be determined by the explicit solution of the perturbed continuity equation (2.20) remaining consistent with the AC method [3] as

$$
P^{(n)}=P^{(n-1)}-\Delta \tau \beta(\nabla \cdot \mathbf{U})^{(n)},
$$

therefore there is no theoretical barrier to drop out the pressure gradient term from the momentum equation (2.6) as in the classical FS-PP method of Chorin [5]. Thus the first step of the FSAC-PP algorithm is to estimate an inital pressure field via the explicit solution of the perturbed continuity equation (2.24) in each pseudo-time step. In this way, the 
convective flux terms $\nabla \cdot(\tilde{\mathbf{U}} \otimes \tilde{\mathbf{U}})^{(n)}$ can be recovered by employing a CB Godunov-type scheme (2.22) with the inclusion of high-order intercell flux interpolations and the solution of the local Riemann problem at pseudo-time level $n$. For the velocity components, the FSAC-PP approach retains the inviscid CB Godunov-type counterpart of the hyperbolic AC formulation (2.22), but the characteristic computation of the pressure gradient term relying on Eq. (2.8) is excluded from the dual-time stepping procedure.

After estimating an initial pressure field $P^{(n)}$ relying on the explicit solution of the perturbed continuity equation (2.24) and computing the right hand side of the fractional momentum equation (2.21), an intermediate velocity field can be estimated in each pseudotime step by remaining consistent with the FS-PP method [5] as

$$
\hat{\mathbf{U}}=\mathbf{U}^{(n)}+\Delta \tau\left[-\frac{\partial \mathbf{U}}{\partial t^{*}}-\nabla \cdot(\tilde{\mathbf{U}} \otimes \tilde{\mathbf{U}})+\frac{1}{R e} \nabla^{2} \mathbf{U}\right]^{(n)},
$$

where the Laplacian viscous flux terms can be discretized by employing second- or higherorder accurate central approximations. In the present work, a second-order central difference scheme is employed for the viscous flux terms.

The temporal accuracy of the pseudo-time marching for solving the governing equations (2.20)-(2.21) can be improved further by applying an explicit Runge-Kutta pseudotime integration scheme $[20,40]$. In this paper, the first-order temporal accuracy of the pseudo-time marching is advanced by employing an explicit fourth-order scheme, thus improving the solution of the intermediate velocity field [1] as

$$
\left\{\begin{array}{l}
\hat{\mathbf{U}}_{R K, 1}=\hat{\mathbf{U}}_{R K}^{(n)} \\
\hat{\mathbf{U}}_{R K, 2}=\hat{\mathbf{U}}_{R K}^{(n)}+\frac{\Delta \tau}{2} \operatorname{RHS}\left(\hat{\mathbf{U}}_{R K, 1}\right), \\
\hat{\mathbf{U}}_{R K, 3}=\hat{\mathbf{U}}_{R K}^{(n)}+\frac{\Delta \tau}{2} \operatorname{RHS}\left(\hat{\mathbf{U}}_{R K, 2}\right), \\
\hat{\mathbf{U}}_{R K, 4}=\hat{\mathbf{U}}_{R K}^{(n)}+\Delta \tau \operatorname{RHS}\left(\hat{\mathbf{U}}_{R K, 3}\right), \\
\hat{\mathbf{U}}_{R K}=\hat{\mathbf{U}}_{R K}^{(n)}+\frac{\Delta \tau}{6}\left[\sum_{i=1,4} \operatorname{RHS}\left(\hat{\mathbf{U}}_{R K, i}\right)+2 \sum_{i=2,3} \operatorname{RHS}\left(\hat{\mathbf{U}}_{R K, i}\right)\right],
\end{array}\right.
$$

where the $\hat{\mathbf{U}}_{R K}=\left[\begin{array}{llll}P / \beta & \hat{U} & \hat{V} & \hat{W}\end{array}\right]^{T}$ vector stands for the stages of the pseudo-time advancement, and RHS $\left(\hat{\mathbf{U}}_{R K}\right)$ represents the right hand side of the system of governing equations (2.20)-(2.21) in each integration step.

After performing a pseudo-time advancement to estimate a highly accurate intermediate velocity field $\hat{\mathbf{U}}$, the pressure field is updated in each pseudo-time step by taking into account the incompressibility constraint, $\nabla \cdot \mathbf{U}^{(n+1)}=0$. In the dual-time stepping procedure via pseudo-time splitting, the divergence of the

$$
\frac{\mathbf{U}^{(n+1)}-\hat{\mathbf{U}}}{\Delta \tau}=-\nabla P^{(n+1)}
$$


semi-discrete equation is a second-order elliptical pressure-Poisson equation $[5,13]$ as

$$
\nabla^{2} P^{(n+1)}=\frac{1}{\Delta \tau} \nabla \cdot \hat{\mathbf{U}}
$$

the pressure field is thus approximated by solving implicitly a pressure-Poisson equation in each pseudo-time step instead of the characteristic Godunov-type reconstruction of the pressure gradient term in the momentum equation (2.6). The initial values for the implicit solution of a pressure-Poisson equation (2.28) are obtained by the explicit solution of the perturbed continuity equation (2.24) to provide the consistency of the solution. Similarly to the FS-PP method [5], it is assumed that the implicit solution of the pressure-Poisson equation (2.28) provides an approximately divergence-free velocity field at each pseudotime step. Therefore it is expected that the pseudo-pressure term will gradually tend to be zero within a very small threshold value in the perturbed continuity equation (2.20).

The velocity field is updated along the characteristics via the implicit solution of the pressure-Poisson equation (2.28) in each pseudo-time step as

$$
\mathbf{U}^{(n+1)}=\hat{\mathbf{U}}-\Delta \tau \nabla P^{(n+1)} .
$$

In order to satisfy the continuity equation (2.3), the FSAC-PP procedure at each pseudotime step has to be repeated until the pseudo-pressure term in the perturbed continuity equation (2.20) and the pseudo-time derivatives in each scalar fractional momentum equation approach very small values (near the machine-zero in computing terms) [1]. In other words, when the continuity equation (2.3) is satisfied, the pseudo-pressure term in the perturbed continuity equation (2.20) becomes equal to a very small threshold value defined by the user as the machine-zero value. For the steady-state solution, the convergence criterion in each pseudo-time step is defined by

$$
\max \left(\beta\left|\nabla \cdot \mathbf{U}^{(n+1)}-\nabla \cdot \mathbf{U}^{(n)}\right|,\left|\frac{\mathbf{U}^{(n+1)}-\mathbf{U}^{(n)}}{\Delta \tau}\right|\right) \leq \varepsilon .
$$

When this criterion is satisfied, the continuity equation (2.3) will also be satisfied. Note that the perturbed continuity equation (2.20) is also appropriate for controlling the pseudotime stepping procedure, because when the right hand side of Eq. (2.20) becomes equal to zero, the steady-state solution of the system is achieved. Due to the velocity field update along the characteristics at each pseudo-time step, the difference between the divergence of the velocity fields in pseudo-time levels $n+1$ and $n$ will decrease. Therefore

$$
\max \left(\beta\left|\nabla \cdot \mathbf{U}^{(n+1)}-\nabla \cdot \mathbf{U}^{(n)}\right|\right) \leq \varepsilon
$$

is a strict criterion providing the compatibility with the classical AC method [1], hence satisfying the perturbed continuity equation (2.5). Since the continuity equation (2.3) is satisfied iteratively due to the PP step and velocity field update along the characteristics 
at each pseudo-time step, the pseudo-pressure term in the perturbed continuity equation (2.20) will tend to be zero step by step. Note that it is necessary to perform sufficient sub-iterations for the implicit solution of the pressure-Poisson equation (2.28) to avoid possible oscillations in the pressure field solution. For unsteady flows, when the steadystate solution is achieved in the pseudo-time stepping process, the solution has also to be advanced in real-time in order to continue the dual-time stepping procedure $[20,21]$.

As a summary, the proposed FSAC-PP unified algorithm consists of seven main steps (see the flowchart of the algorithm in Fig. 1):

1. The perturbed continuity equation (2.20) is solved explicitly to estimate an initial pressure field in each pseudo-time step of the dual-time stepping procedure by remaining consistent with the AC method [3]. The pressure and velocity fields are equal to the initial values of the problem at the first real-time step $\left(t^{*}=0\right)$.

2. The Godunov-procedure is performed to compute the CB Godunov-type convective velocity flux vector (2.22) and the right hand side of the fractional momentum equation (2.21) relies on the pressure field estimated in Step 1. The intercell primitive variables can be approximated by applying first-, second-, third- or even higher-order interpolations (e.g. fifth- and ninth-order WENO schemes [24-26]). We note that any appropriately chosen order of interpolation can be employed.

3. An intermediate velocity field (2.25) is estimated via pseudo-time splitting in conjunction with a CB Godunov-type scheme computed in Step 2 by dropping out the characteristic pressure gradient term from the dual-time stepping procedure.

4. The temporal accuracy of the pseudo-time marching process is improved further by applying an appropriately chosen explicit (e.g. fourth-order) Runge-Kutta pseudo-time integration scheme to estimate a high-accurate intermediate velocity field.

5. A pressure-Poisson equation (2.28) is solved implicity to update the pressure field instead of the characteristic computation of the pressure gradient term in the momentum equation. The initial values for the implicit solution of a pressure-Poisson equation (2.28) are obtained in each pseudo-time step by the explicit solution of the perturbed continuity equation (2.20) to provide the consistency of the numerical solution with the classical FS-PP method $[5,6]$.

6. The velocity field is updated along the characteristics via the implicit solution of the pressurePoisson equation (2.28) in each pseudo-time step.

7. For unsteady flows, when the steady-state solution is achieved in the pseudo-time stepping process, the solution is advanced in real-time. Explicit time-marching schemes are recommended from first- up to higher-orders of accuracy.

\subsection{Pseudo-time stepping strategy}

The discretized system of governing equations is solved via a dual-time stepping numerical procedure, therefore a pseudo-time stepping strategy is required for stability reasons. The accuracy and convergence properties of $\mathrm{CB}$ schemes were investigated by 


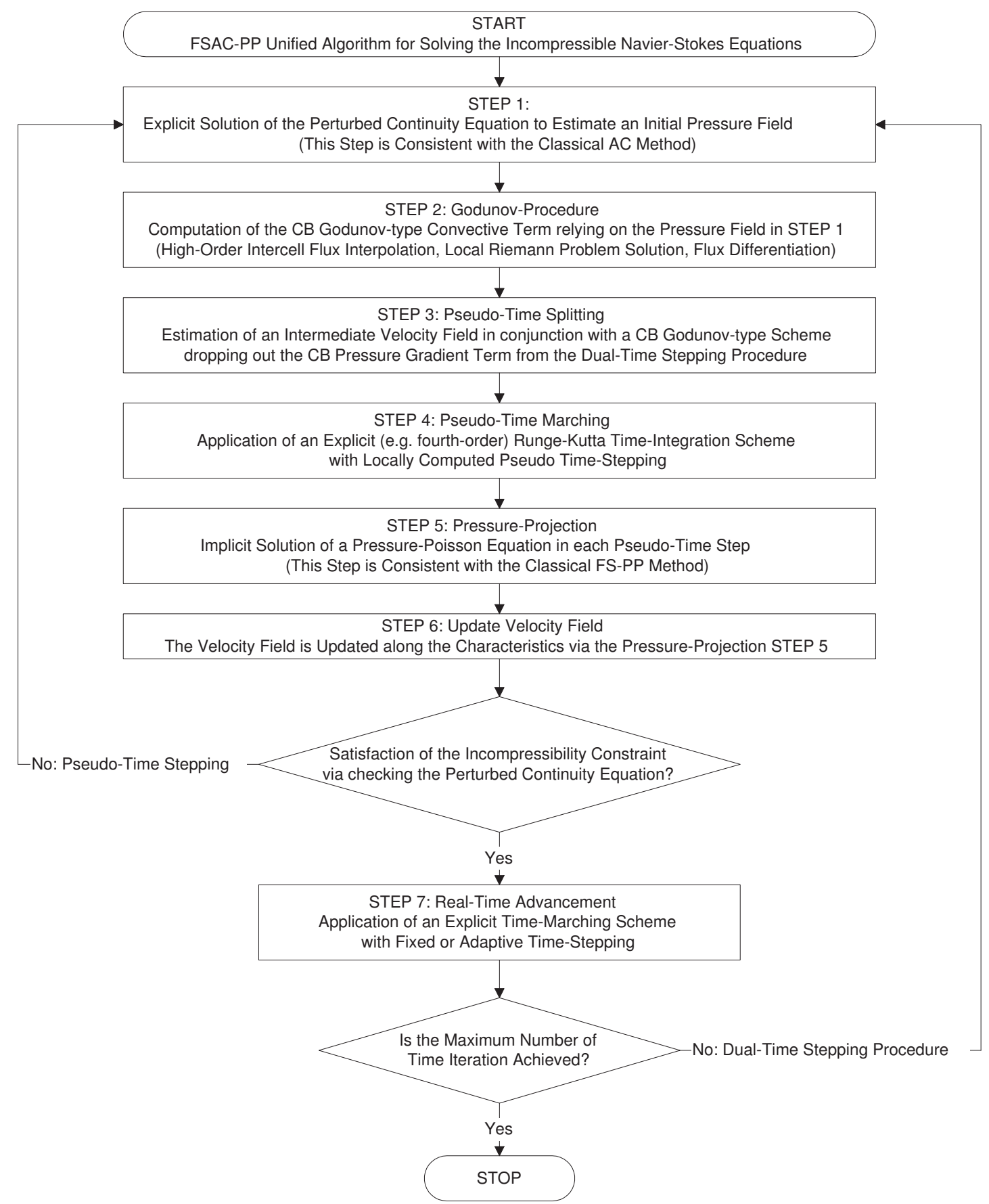

Figure 1: Flowchart of the proposed FSAC-PP unified algorithm for incompressible flows. 
Shapiro and Drikakis [22] based on an eigenvalue analysis to estimate the locally computed pseudo-time step size. The relationship between the pseudo-time step and characteristic condition numbers provides the stability of the numerical procedure [20] as

$$
\begin{aligned}
\Delta \tau_{i, j, k}^{\text {Inviscid }} & =\frac{\text { CFL }_{\text {Inviscid }}}{\left[\max _{\mathrm{m}=1-4}\left(\left|\lambda_{1}\right|,\left|\lambda_{2}\right|\right)_{m}\right]_{i, j, k}}, \\
\Delta \tau_{i, j, k}^{\text {Viscous }} & =\frac{\mathrm{CFL}_{\text {Viscous }} R e}{4\left[\max _{\mathrm{n}=1,2,3}\left(d l_{n}\right)\right]_{i, j, k}},
\end{aligned}
$$

where $\mathrm{CFL}_{\text {Inviscid }}$ and $\mathrm{CFL}_{\text {Viscous }}$ are the Courant-Friedrichs-Lewy (CFL) numbers of the inviscid and viscous fluxes, respectively, and $d l_{n}$ represents the cell dimension according to the coordinate directions. The CFL numbers are characteristic condition numbers relating to the stiffness of the numerical solution [22]. For stability reasons, the locally computed pseudo-time step is defined by the minimum value of the locally determined pseudo-time steps for the inviscid and viscous fluxes as

$$
\Delta \tau_{i, j, k}=\gamma \cdot \min \left(\Delta \tau_{i, j, k}^{\text {Inviscid }}, \Delta \tau_{i, j, k}^{\text {Viscous }}\right),
$$

where $\gamma \in] 0,1]$ is an appropriately chosen safety factor to ensure numerical stability [41]. The pseudo-time step depends on the CFL numbers and the eigenvalues, which in turn depend on the AC parameter $\beta$. These parameters have to be adjusted for each computational test case to ensure the stability and convergence of the numerical solution $[4,42]$.

\subsection{Boundary condition for the pressure}

In the classical FS-PP method, a Neumann-type boundary condition was used by Chorin [5] and Perot [13], which can be written as

$$
\frac{\partial P^{(n+1)}}{\partial n}=0,
$$

and it is also called an "inviscid-type boundary condition" for the pressure [43, 44]. Karniadakis et al. [45] introduced a consistent Neumann-type boundary condition for the pressure field using a spectral element discretization employing a high-order timesplitting method for solving unsteady, incompressible flow problems. They pointed out that the inviscid-type boundary condition (2.35) for the pressure is valid at high Reynolds number flows, but not for low Reynolds number microflows, because this condition leads to a divergence error at the boundary [43]. For microflows by using the dimensionless form of the governing equations, the following consistent boundary condition is suggested by Karniadakis et al. [43, pp. 514-517] as

$$
\frac{\partial P^{(n+1)}}{\partial n}=\frac{1}{R e} \mathbf{n} \nabla^{2} \mathbf{U}^{(n+1)}-\mathbf{n} \hat{\mathbf{U}},
$$


where $\mathbf{n}$ is the normal vector, which is perpendicular to the surface with outward direction. The Neumann-type boundary condition for the pressure prescribed at all boundaries introduces a difficulty; in particular the coefficient matrix of the system of linear equations of the discretized pressure-Poisson equation becomes singular. To overcome this problem, the pressure value has to be prescribed at least at one point of the domain as an additive constant [46]. According to Guermond et al. [47] among others, the inviscid Neumann-type boundary condition is not a physical condition for the pressure, but they do consider this boundary condition to be mathematically correct for the pressurePoisson equation. Bell et al. [8] and Guermond et al. [47] also pointed out, as a consequence of Ladyzhenskaya's [7] orthogonality theorem, that there is no need for a boundary condition for the pressure because it makes the system over-determined [8]. For more details about the orthogonality theorem of Ladyzhenskaya and the orthogonality of the pressure gradient with a solenoidal vector field see [7].

\section{Validation of the FSAC-PP method for benchmark problems}

An in-house FORTRAN 90/95 code has been developed for solving the stationary and unsteady, incompressible Navier-Stokes equations relying on the proposed FSAC-PP solution method [1]. For benchmark test cases, the validation of this unified approach, in conjunction with the further extended version of the non-linear FMG-FAS three-level multigrid algorithm [18], is presented in this Section. The numerical results were compared to other methods in terms of accuracy and efficiency.

For stationary, incompressible, constant density flows at (very) low Reynolds numbers, a two-dimensional pressure-driven laminar flow was simulated in a straight microfluidic channel. The numerical investigation of this fluid flow problem is an appropriate example for the validation of low Reynolds number flow solvers for microfluidic applications, because an analytical solution exists for the fully-developed laminar outlet velocity profile. Thus, the computational results can be compared to analytical solutions and the original AC and PP methods at different low Reynolds numbers, taking into account extremely low Reynolds number cases, e.g., from $10^{-1}$ up to $10^{-4}$ (see more details about pressure-driven laminar flows in [48, pp. 99-122], and in [49-51]).

The FSAC-PP method was also validated for stationary laminar flows in a lid driven square cavity at moderate Reynolds numbers ( $R e=100,400$, and 1000). For this twodimensional problem, the numerical results were compared to the AC and PP methods, and the classical computational data of Ghia et al. [52].

For stationary simulation test cases, the convergence properties and numerical errors of each employed interpolation scheme were demonstrated in conjunction with the FMG-FAS multigrid algorithm [18]. The computational times were presented at different Reynolds numbers. The numerical convergence of the AC, PP and FSAC-PP methods was measured by using the maximum value of the fourth-order Runge-Kutta residuals 
of the pseudo-time stepping procedure, which can be defined by

$$
\max \left(\left|\frac{\mathbf{U}_{R K}^{(n+1)}-\mathbf{U}_{R K}^{(n)}}{\Delta \tau}\right|\right) \leq \varepsilon,
$$

where the $\mathbf{U}_{R K}=\left[\begin{array}{llll}P / \beta & U & V & W\end{array}\right]^{T}$ vector represents the numerical solution of the governing equations between two pseudo-time levels. The pseudo-time stepping iterations of the AC, PP and FSAC-PP methods were carried out until a steady-state solution was achieved; this means that the Runge-Kutta residuals did not change significantly below a very small $\varepsilon$ threshold defined by the user as the machine-zero value.

To measure the computational time and examine the capability of the FSAC-PP approach at a higher Reynolds number, an unsteady, incompressible, turbulent flow was tested in a lid driven cubical cavity at $R e=10000$. For this three-dimensional problem, experimental data are available in the paper of Prasad and Koseff [53]. It is important to note that several authors have used this benchmark to verify their computer codes and validate their numerical methods (see, e.g., $[12,54-56])$. The turbulent flow simulations were performed by using the Implicit Large Eddy Simulation (ILES) method in conjunction with the AC, PP, and the unified FSAC-PP algorithms.

\subsection{Laminar flow in a straight microfluidic channel at low $R e$ numbers}

For steady-state, incompressible, constant density flow in a straight microfluidic channel, according to the analytical solution for the one-dimensional case, the axial velocity component depends on the coordinate of the radial direction $u=u(y)$ while the other velocity components vanish $(v=w=0)$. It is known from the literature that the AC method is efficient at Reynolds numbers from 1 up to $10^{3}[2,4]$. The reasons for this are the restriction for the locally computed pseudo-time step in Eq. (2.34) and the choice of the AC parameter in Eq. (2.10) which can lead to a slow convergence rate at $R e \ll 1$. For microfluidic applications, the typical Reynolds numbers range from $10^{-1}$ up to $10^{2}$ (see, e.g., [21]), therefore it was pertinent to validate the FSAC-PP method at very low Reynolds numbers. For a laminar flow in a channel, the Reynolds number can be defined by

$$
R e=\frac{2 h \tilde{u}}{v},
$$

where $h$ is the height of the microfluidic channel, $\tilde{u}$ is the average velocity, and $v$ is the kinematic viscosity of the fluid. From wall to wall, the fully-developed stationary velocity distribution [57, p. 10] can be derived as

$$
u(y)=\rho \frac{\Delta \Pi}{2 \mu l} y(h-y),
$$

where $\rho$ is the density of the fluid, $\mu$ is the dynamic viscosity of the fluid, $l$ is the length of the microfluidic channel, and $\Pi$ is the total potential proportional to the pressure dif- 
ference between the inlet and the outlet sections as

$$
\Delta \Pi=\frac{\Delta p}{\rho} .
$$

The characteristic quantities can also be computed such as the volume flow rate which is

$$
Q=w_{c} \int_{0}^{h} u(y) d y=w_{c} \frac{\Delta \Pi}{2 v l} \int_{0}^{h} y(h-y) d y=w_{c} \frac{\Delta \Pi}{12 v l} h^{3},
$$

where $w_{c}$ is the width of the microfluidic channel, and the average velocity can be expressed by the ratio of volume flow rate to cross-sectional area as

$$
\tilde{u}=\frac{Q}{A}=\frac{\Delta p}{12 \mu l} h^{2}
$$

where the pressure difference between the inlet and outlet sections is written as

$$
\Delta p=\frac{12 \mu l}{h^{2}} \tilde{u} .
$$

By using Eqs. (3.2)-(3.7), all physical quantities of the pressure-driven laminar flow can be computed analytically at the outlet cross-section of the straight microfluidic channel.

\subsubsection{The straight microfluidic benchmark channel}

The computational domain of the straight microfluidic channel and the contour lines of the horizontal velocity component in the fully developed pressure-driven laminar flow are shown in Fig. 2. The numerical grid consists of $181 \times 41$ node points $(180 \times 40)$ control cells on the fine grid. The height $h$ of the microfluidic channel is $10 \mu \mathrm{m}$, and the length $l$ is equal to $100 \mu \mathrm{m}$. Dimensionless quantities were used in the simulations, therefore the spatial coordinates were normalized by the height $h$ of the channel.

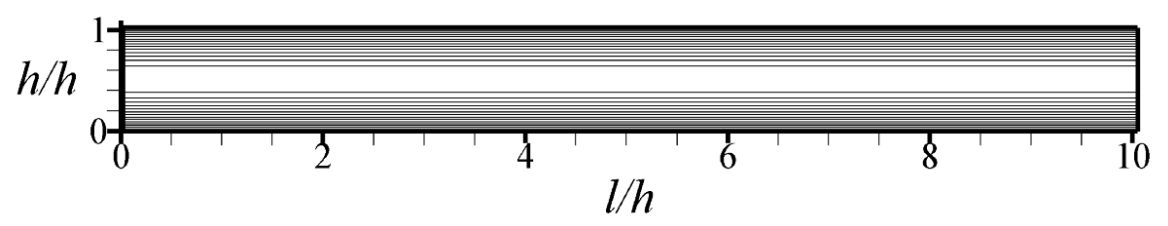

Figure 2: Computational domain and contour lines of the horizontal velocity component in the fully developed pressure-driven laminar flow in a straight microfluidic channel by using dimensionless spatial coordinates.

A mathematical task can be formulated in terms of boundary and initial conditions for the pressure-driven laminar flow in the straight microfluidic channel. From wall to wall, the inlet velocity profile was normalized by the average flow velocity $\tilde{u}$ and defined as

$$
U_{\text {inlet }}(0, Y)=\frac{u}{\tilde{u}}=6 Y(1-Y) \text {, }
$$


where $u_{*}$ is the wall-friction velocity [48]. The dimensionless pressure was prescribed equally to be zero at the inlet section, which can be written as $P_{\text {inlet }}(0, Y)=0$. Thus the boundary conditions were the Dirichlet-type in the inlet section. The outlet boundary conditions for the dimensionless velocity components can be prescribed as

$$
\frac{\partial U(L, Y)}{\partial X}=0 \quad \text { and } \quad \frac{\partial V(L, Y)}{\partial X}=0
$$

which were Neumann-type boundary conditions, where $L$ is the dimensionless spatial coordinate of the outlet section. The pressure was prescribed equally to be one at the outlet section, and can be written as $P_{\text {outlet }}(L, Y)=1$. The velocity components $U$ and $V$ were equal to zero on the bottom and upper walls as

$$
U_{\text {upper wall }}(X, 1)=U_{\text {bottom wall }}(X, 0)=V_{\text {upper wall }}(X, 1)=V_{\text {bottom wall }}(X, 0)=0 .
$$

Neumann-type and consistent boundary conditions were prescribed for the pressure on the bottom and upper walls, as suggested by Karniadakis et al. [43, pp. 514-517] based on Eq. (2.36). The initial conditions for the dimensionless velocity components $U, V$ and the pressure values $P$ were equal to zero in each interior cell.

\subsubsection{Validation of the FSAC-PP method at very low Re numbers}

For the validation of the FSAC-PP method, compared to the AC and PP methods, and an analytical solution for stationary flows from $R e=10$ to $10^{-4}, 216$ simulations were performed by using three different methods with three different intercell flux interpolation schemes on four different girds at six different Reynolds numbers.

As discussed above, for computing the convective fluxes, a transport CB Godunovtype scheme (2.22) is employed for the AC and FSAC-PP methods, because a) the CB Godunov-type scheme can be derived from the hyperbolic system of the AC formulation and $b$ ) the FSAC-PP method unifies the AC method with the FS-PP method via pseudotime splitting. Due to the elliptical nature of the FS-PP method, the Lax-Friedrichs scheme was employed for those simulations when the PP method itself was used. The accuracy of the pseudo-time stepping procedure was improved further by applying an explicit fourth-order Runge-Kutta integration scheme [1,17,20].

The non-linear FMG-FAS multigrid technique [18] was used to accelerate the numerical procedure by using 100 pre- and post-smoothing iterations on the fine grid, 10 preand post-smoothing iterations on the medium grid, and 10 iterations on the coarse grid. Note that the choice of the number of pre- and post-relaxation steps is dependent on the fluid flow case [21, p. 616]. The simulation parameters including the characteristic condition numbers are summarized in Table 1.

The grid convergence study is an indispensable part of a validation procedure for computational fluid dynamics (CFD) methods. In this test case, four different grid levels with $G 1=200, G 2=800, G 3=3200, G 4=7200$ cells are used for validating the proposed 
Table 1: Simulation parameters of six numerical test cases for the $A C$ parameter $\beta$, for the $C F L_{i n v}$ and $C F L_{v i s}$ Courant-Friedrichs-Lewy numbers associated with the inviscid and viscous fluxes at various Reynolds numbers Re.

\begin{tabular}{||c|c|c|c|c|c|c||}
\hline \multirow{3}{*}{} & \multicolumn{7}{|c||}{ Reynolds Numbers $(\mathrm{Re})$} \\
\cline { 2 - 7 } & 10 & 1 & 0.1 & 0.01 & 0.001 & 0.0001 \\
\cline { 2 - 7 } & \multicolumn{6}{|c||}{ Simulation Parameters } \\
\hline$\beta$ & 10 & $10^{2}$ & $10^{3}$ & $10^{4}$ & $10^{5}$ & $10^{6}$ \\
\hline$C F L_{\text {inv }}$ & 0.2 & 0.05 & 0.015 & 0.015 & 0.015 & 0.015 \\
\hline$C F L_{\text {vis }}$ & 0.15 & 0.05 & 0.015 & 0.015 & 0.015 & 0.015 \\
\hline
\end{tabular}

Table 2: Grid refinement ratios $r$ and the order of truncation errors $p$ on different grids.

\begin{tabular}{||c|c|c||}
\hline Grid & Grid Refinement Ratio $r$ & Orders of Truncation Error $p$ \\
\hline G1 $\div$ G2 & 4 & $1,2,3$ \\
\hline G2 $\div$ G3 & 4 & $1,2,3$ \\
\hline G3 $\div$ G4 & 2.25 & $1,2,3$ \\
\hline
\end{tabular}

FSAC-PP method compared to the AC and PP methods and an analytical solution, respectively. The grid refinement ratio $r$ and the order of the intercell flux interpolation schemes relating to the truncation error $p$ are summarized in Table 2.

The results showed that grid convergence was observed in most cases of the AC, PP and FSAC-PP methods from $R e=10$ to 0.1 based on the simulation parameters at different grid levels (see Figs. 4-6). By decreasing the Reynolds number, grid convergence was observed only in most cases of the PP and FSAC-PP methods from $R e=0.01$ to 0.0001 . The results did not show significant changes when using more than $180 \times 40$ control cells on the domain for this microfluidic benchmark problem.

The characteristic condition numbers as $C F L_{i n v}$ and $C F L_{v i s}$ had to be decreased and the AC parameter $\beta$ had to be increased when the Reynolds number was decreased from 10 to 0.0001 . Small CFL numbers led to very small values of the locally computed pseudotime step, and the large values of the $\mathrm{AC}$ parameter $\beta$ ensured the numerical convergence (see Table 1). The computational efficiency of the AC, PP and FSAC-PP methods was measured based on the required number of multigrid cycles $N_{m g}$ until the lowest maximum Runge-Kutta residual $R K_{\text {maxres }}$ was achieved on the fine grid.

The convergence histories of each simulation are summarized in Tables 4-9, and in Fig. 7. The results showed that the FSAC-PP method required the lowest number of multigrid iterations $N_{m g}$ on the fine grid compared to the AC and PP methods for this microfluidic benchmark test case. The computational times are summarized in Table 3 at three different Reynolds numbers on a Dual-Core E5300 2.60 GHz computer with 8GB RAM. For stationary flows, it was observed that the inclusion of the PP step in the pseudo-time stepping procedure of the $\mathrm{AC}$ formulation can improve the convergence properties of the FSAC-PP algorithm, compared to the AC and FS-PP methods. The reason is that when the FSAC-PP algorithm is used, it is sufficient to perform a small number 
Table 3: Computational times for a stationary flow problem in a straight microfluidic channel at $\operatorname{Re}=10,1$, and 0.1 on a Dual-Core E5300 $2.60 \mathrm{GHz}$ computer by employing a $3^{\text {rd }}$-order interpolation scheme for the convective term.

\begin{tabular}{||c|c|c|c||}
\hline \multirow{2}{*}{} & \multicolumn{3}{|c||}{ Reynolds Numbers $(\mathrm{Re})$} \\
\cline { 2 - 4 } & 10 & 1 & 0.1 \\
\hline Solution Methods & \multicolumn{3}{|c||}{ Computational Times (Minutes) } \\
\hline AC & 41 & 214 & 1156 \\
\hline PP & 15 & 153 & 473 \\
\hline FSAC-PP & 14 & 102 & 34 \\
\hline
\end{tabular}

of sub-iterations (e.g. between 1 and 10) for solving the pressure-Poisson equation to accelerate the pseudo-time stepping procedure via the perturbed continuity equation.

Numerical solutions to the AC formulation exhibited slow convergence rates irrespective of the values of the AC parameter $\beta$. In the case of the PP method, the rate of convergence was also slow and the number of iterations was the highest, because 1) a small time-step size has to be chosen as, e.g. $\Delta t^{*}=0.001$ or smaller, to achieve a numerically stable solution; and 2) a relatively high number of sub-iterations (e.g. between 100 and 500) was required for solving the pressure-Poisson equation implicitly to obtain accurate results. For these reasons, the FSAC-PP solution method was developed and investigated as an alternative method. When the Reynolds number was decreased, the lowest maximum Runge-Kutta residual was achieved by employing a third-order interpolation scheme; therefore at least accuracy of third-order is recommended for the convective flux terms at (very) low Reynolds numbers (see Table 9 and Fig. 3).

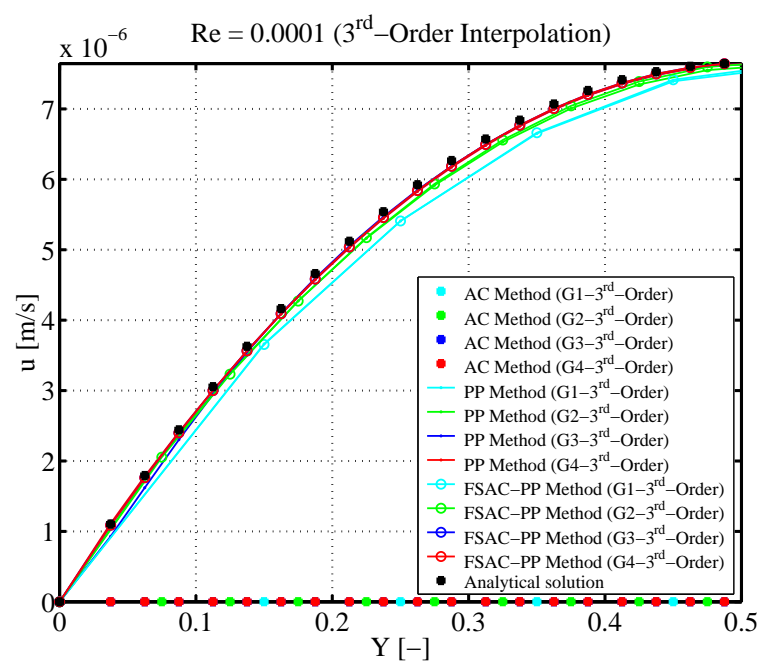

Figure 3: Comparison of analytically and numerically computed outlet velocity profiles relying on the solution of the AC, PP, and FSAC-PP methods in a pressure-driven laminar flow using a $3^{\text {rd }}$-order interpolation scheme at $R e=0.0001[1]$. 
(a) $\operatorname{Re}=10\left(1^{\text {st }}-\right.$ Order Interpolation $)$
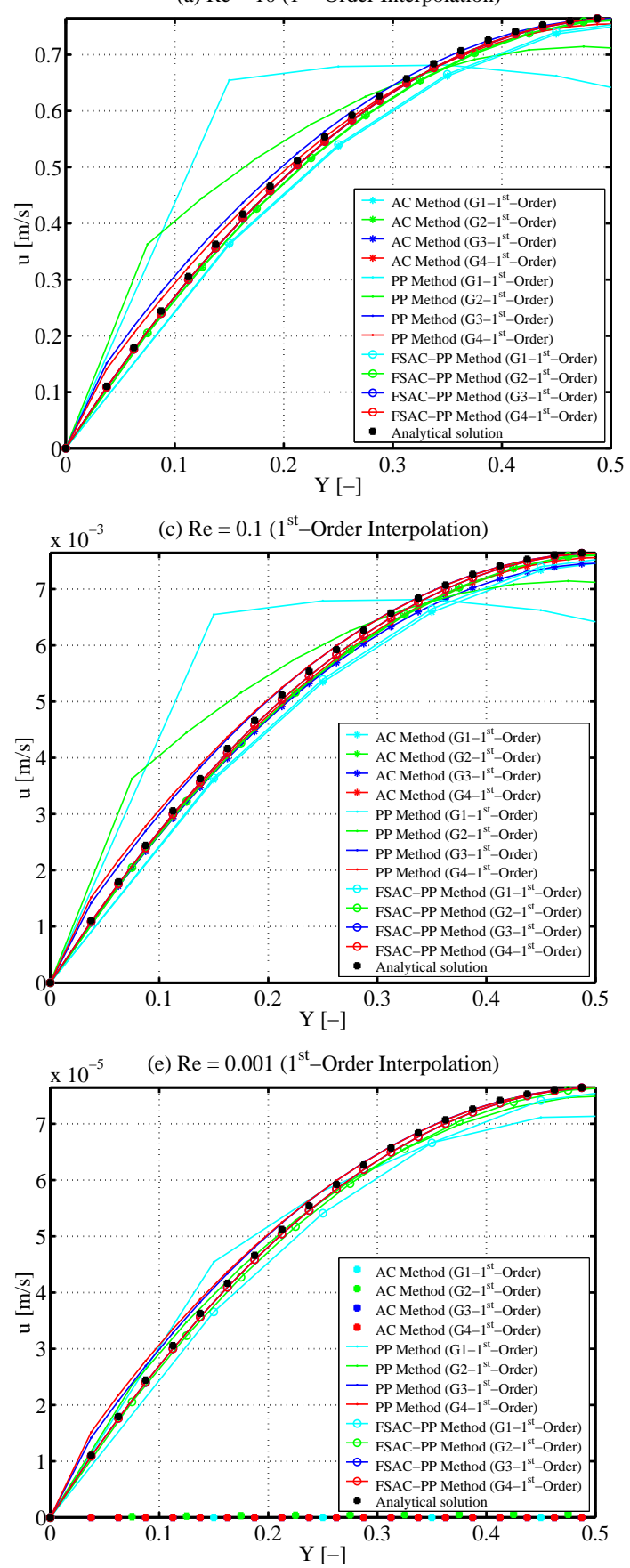

(b) $\operatorname{Re}=1\left(1^{\text {st }}-\right.$ Order Interpolation $)$
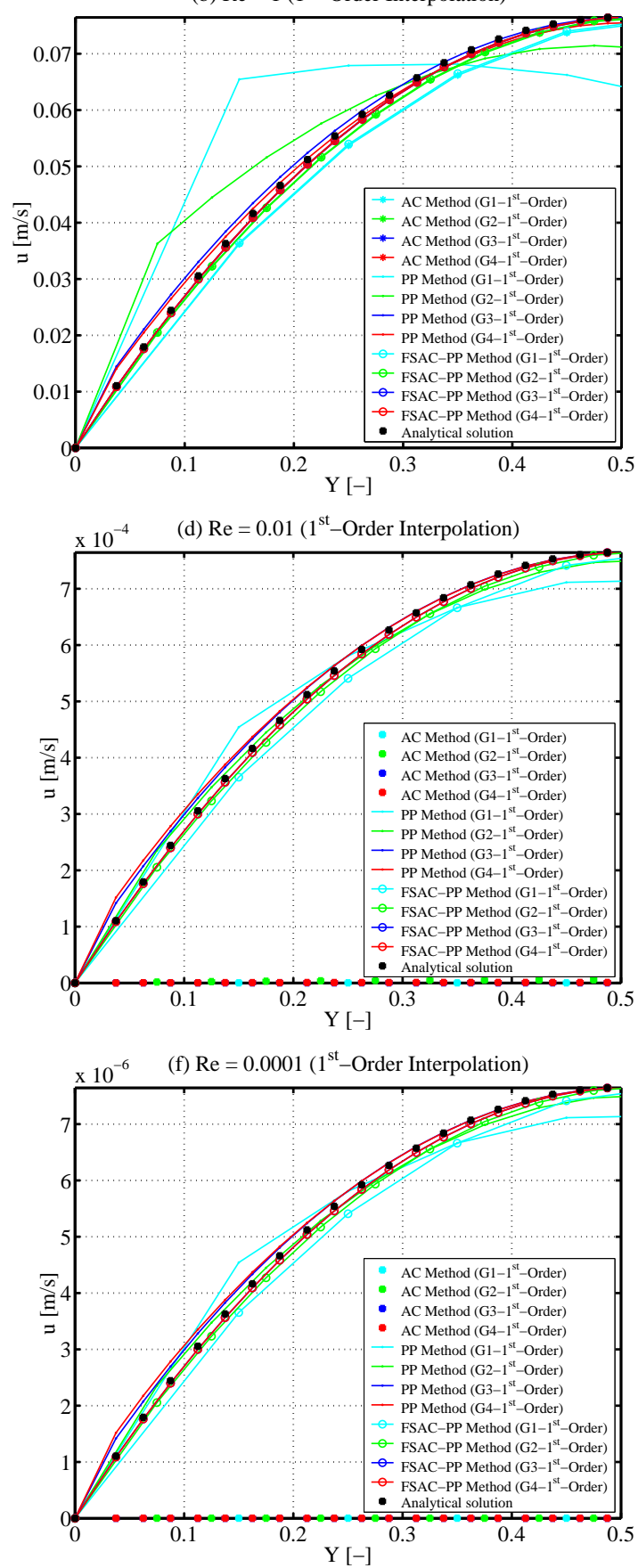

Figure 4: Comparison of analytically and numerically computed outlet velocity profiles relying on the solution of the AC, PP, and FSAC-PP methods in a pressure-driven laminar flow in a straight microfluidic channel using a $1^{\text {st }}$-order interpolation scheme on four different grids at six different Reynolds numbers. 
(a) $\operatorname{Re}=10\left(2^{\text {nd }}-\right.$ Order Interpolation $)$

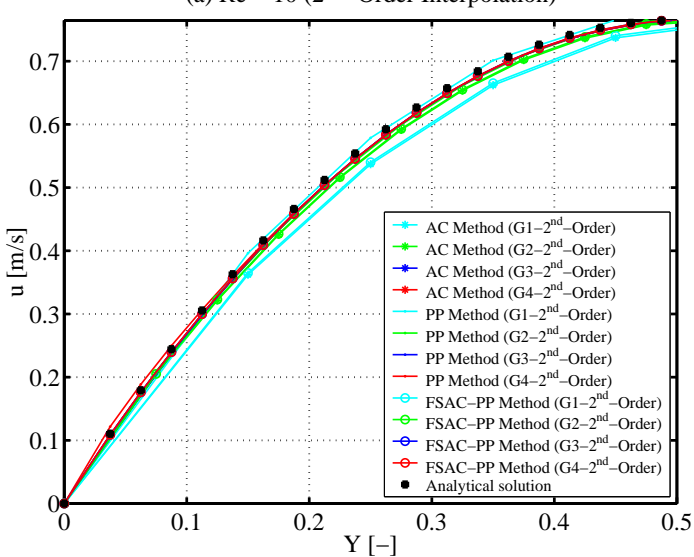

(c) $\operatorname{Re}=0.1\left(2^{\text {nd }}-\right.$ Order Interpolation $)$
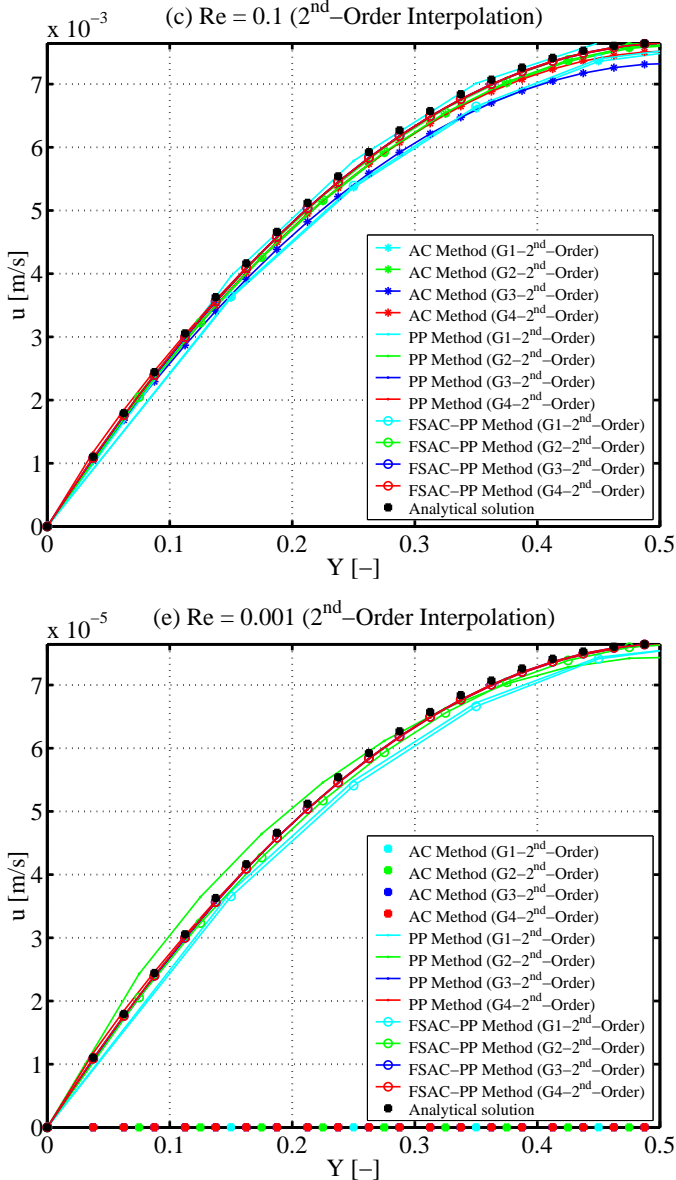

(b) $\operatorname{Re}=1\left(2^{\text {nd }}-\right.$ Order Interpolation $)$

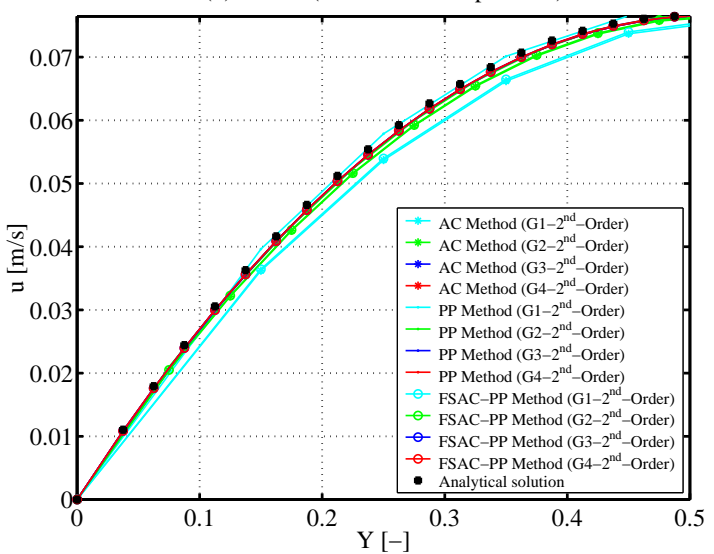

(d) $\operatorname{Re}=0.01\left(2^{\text {nd }}-\right.$ Order Interpolation $)$
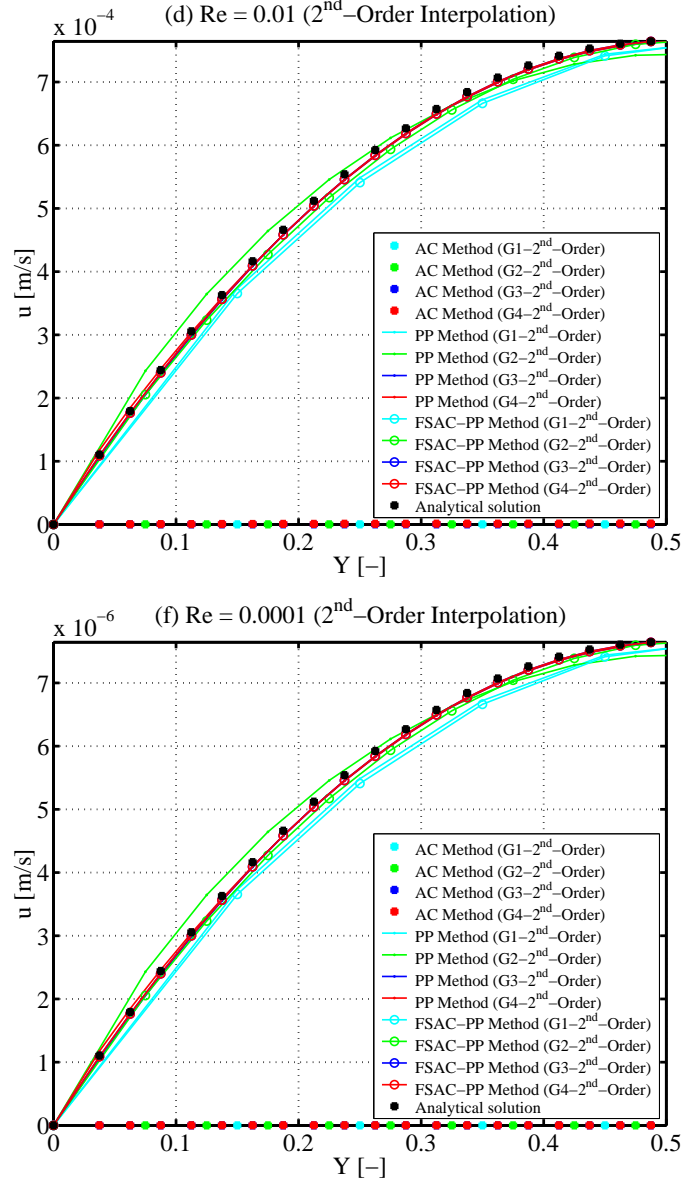

Figure 5: Comparison of analytically and numerically computed outlet velocity profiles relying on the solution of the AC, PP, and FSAC-PP methods in a pressure-driven laminar flow in a straight microfluidic channel using a $2^{n d}$-order interpolation scheme on four different grids at six different Reynolds numbers. 
(a) $\mathrm{Re}=10$ ( $3^{\text {rd }}-$ Order Interpolation $)$
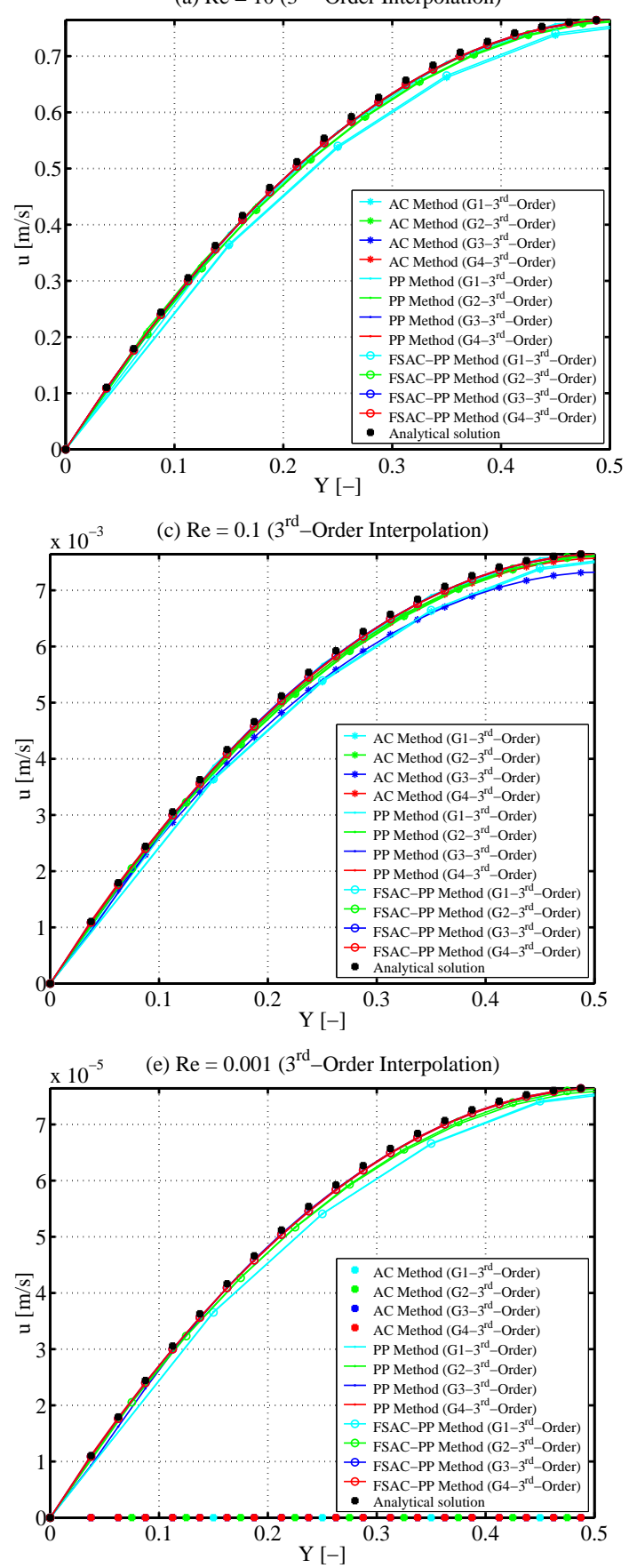

(b) $\operatorname{Re}=1\left(3^{\text {rd }}-\right.$ Order Interpolation $)$
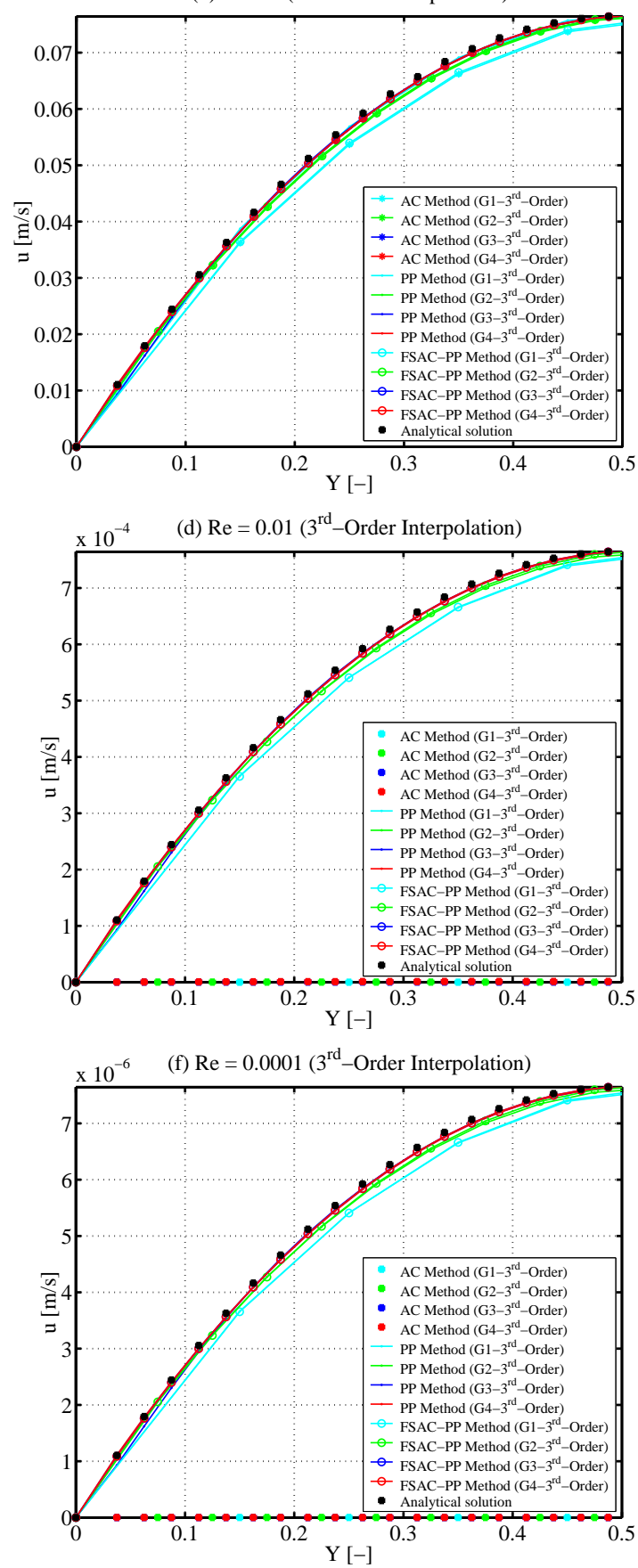

Figure 6: Comparison of analytically and numerically computed outlet velocity profiles relying on the solution of the AC, PP, and FSAC-PP methods in a pressure-driven laminar flow in a straight microfluidic channel using a $3^{r d}$-order interpolation scheme on four different grids at six different Reynolds numbers. 
(a) $\operatorname{Re}=10$

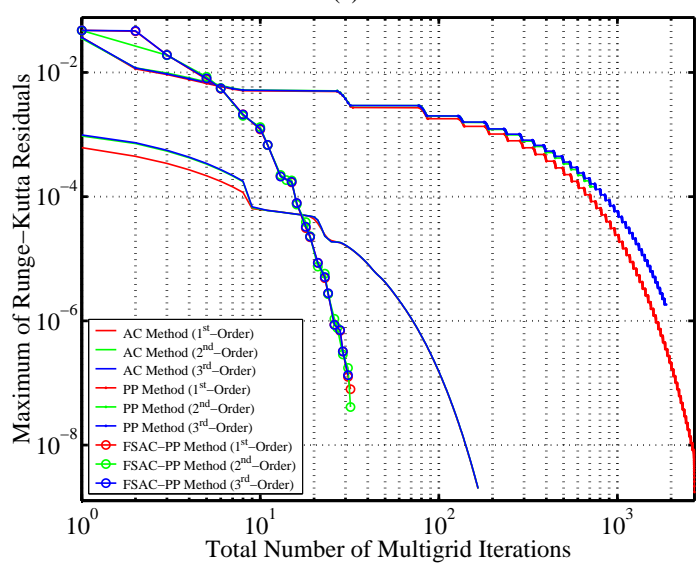

(c) $\operatorname{Re}=0.1$

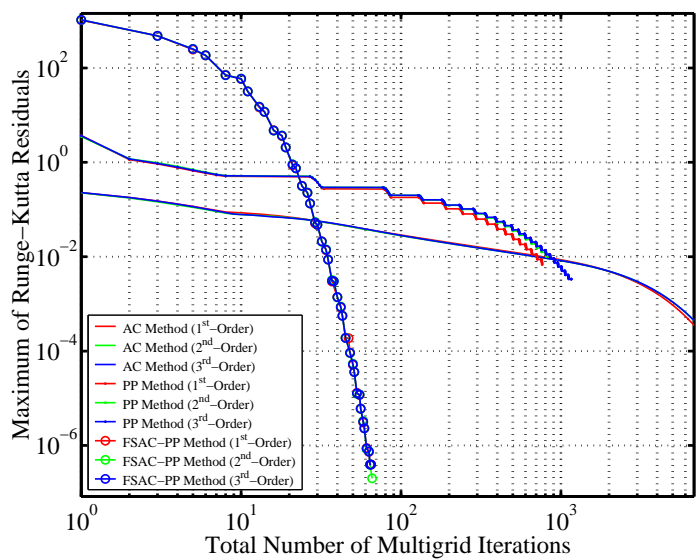

(e) $\operatorname{Re}=0.001$

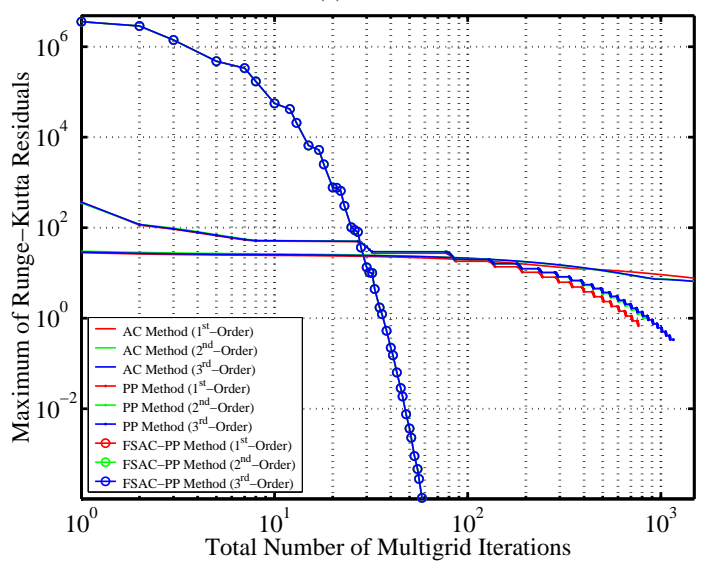

(b) $\operatorname{Re}=1$

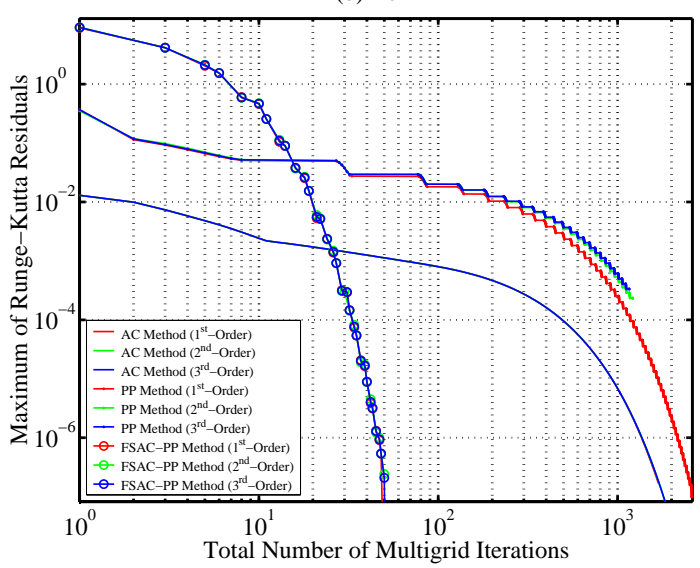

(d) $\operatorname{Re}=0.01$

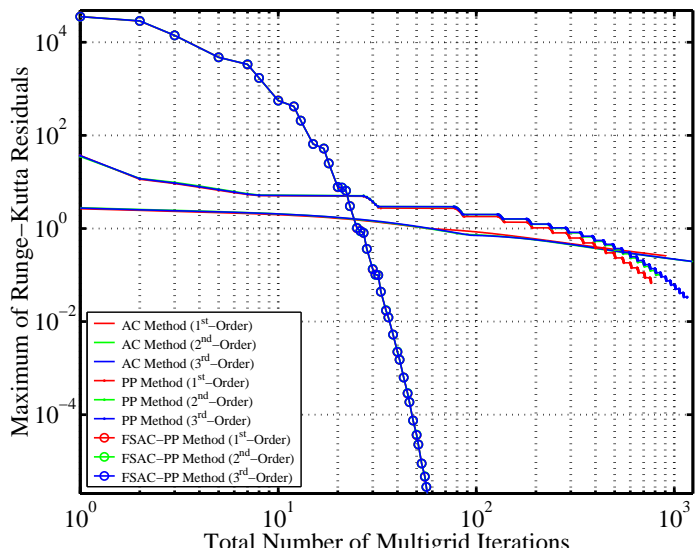

(f) $\mathrm{Re}=0.0001$

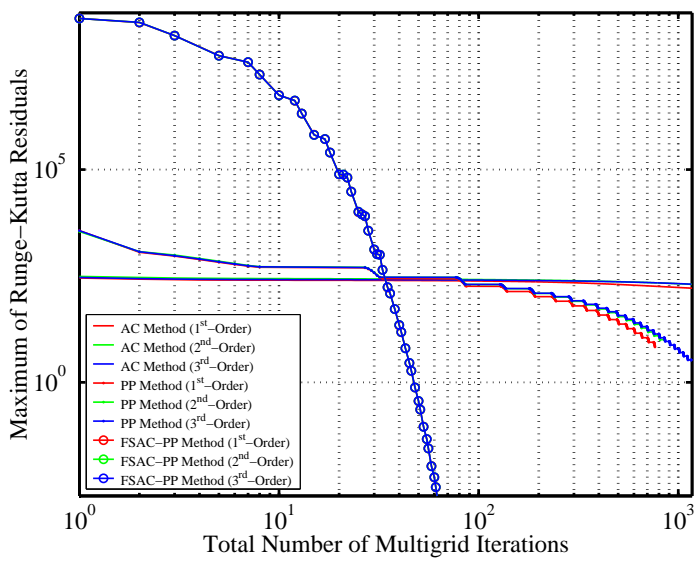

Figure 7: Comparison of the numerical convergence of the AC, PP, and FSAC-PP methods; maximum values of Runge-Kutta residuals versus total number of multigrid iterations on the fine grid at six different Reynolds numbers from $R e=10$ to $R e=0.0001$. 
Table 4: Multigrid iterations and maximum of Runge-Kutta residuals at $R e=10$.

\begin{tabular}{||c|c|c|c||}
\hline \multicolumn{4}{|c||}{ Reynolds Number $(R e=10)$} \\
\hline Intercell Flux Interpolations & Solution Methods & $N_{m g}$ & $R K_{\text {maxres }}$ \\
\hline \multirow{3}{*}{$1^{\text {st }}$ _Order } & $\mathrm{AC}$ & 166 & $0.19995 \cdot 10^{-8}$ \\
\cline { 2 - 4 } & $\mathrm{PP}$ & 2699 & $0.17488 \cdot 10^{-8}$ \\
\cline { 2 - 4 } & $\mathrm{FSAC}-\mathrm{PP}$ & 32 & $0.79310 \cdot 10^{-7}$ \\
\hline \multirow{3}{*}{$2^{\text {nd_Order }}$} & $\mathrm{AC}$ & 166 & $0.19994 \cdot 10^{-8}$ \\
\cline { 2 - 4 } & $\mathrm{PP}$ & 751 & $0.14190 \cdot 10^{-3}$ \\
\cline { 2 - 4 } & $\mathrm{FSAC}-\mathrm{PP}$ & 32 & $0.40928 \cdot 10^{-7}$ \\
\hline \multirow{3}{*}{$3^{\text {rd }}$-Order } & $\mathrm{AC}$ & 166 & $0.19995 \cdot 10^{-8}$ \\
\cline { 2 - 4 } & $\mathrm{PP}$ & 1878 & $0.18354 \cdot 10^{-5}$ \\
\cline { 2 - 4 } & $\mathrm{FSAC}-\mathrm{PP}$ & 31 & $0.13352 \cdot 10^{-6}$ \\
\hline
\end{tabular}

Table 5: Multigrid iterations and maximum of Runge-Kutta residuals at $R e=1$.

\begin{tabular}{||c|c|c|c||}
\hline \multicolumn{4}{|c||}{ Reynolds Number $(R e=1)$} \\
\hline Intercell Flux Interpolations & Solution Methods & $N_{m g}$ & $R K_{\text {maxres }}$ \\
\hline \multirow{3}{*}{$1^{\text {st_Order }}$} & $\mathrm{AC}$ & 1686 & $0.17147 \cdot 10^{-6}$ \\
\cline { 2 - 4 } & $\mathrm{PP}$ & 2610 & $0.98816 \cdot 10^{-7}$ \\
\cline { 2 - 4 } & $\mathrm{FSAC}-\mathrm{PP}$ & 50 & $0.12885 \cdot 10^{-8}$ \\
\hline \multirow{3}{*}{$2^{\text {nd_Order }}$} & $\mathrm{AC}$ & 2548 & $0.19999 \cdot 10^{-8}$ \\
\cline { 2 - 4 } & $\mathrm{PP}$ & 1216 & $0.23133 \cdot 10^{-3}$ \\
\cline { 2 - 4 } & $\mathrm{FSAC}-\mathrm{PP}$ & 50 & $0.24190 \cdot 10^{-6}$ \\
\hline \multirow{3}{*}{$3^{\text {rd_Order }}$} & $\mathrm{AC}$ & 2548 & $0.20027 \cdot 10^{-8}$ \\
\cline { 2 - 4 } & $\mathrm{PP}$ & 1161 & $0.33209 \cdot 10^{-3}$ \\
\cline { 2 - 4 } & $\mathrm{FSAC}-\mathrm{PP}$ & 51 & $0.15745 \cdot 10^{-8}$ \\
\hline
\end{tabular}

Table 6: Multigrid iterations and maximum of Runge-Kutta residuals at $R e=0.1$.

\begin{tabular}{||c|c|c|c||}
\hline \multicolumn{4}{|c||}{ Reynolds Number $(R e=0.1)$} \\
\hline Intercell Flux Interpolations & Solution Methods & $N_{m g}$ & $R K_{\text {maxres }}$ \\
\hline \multirow{3}{*}{$1^{\text {st_-Order }}$} & $\mathrm{AC}$ & 8816 & $0.11695 \cdot 10^{-3}$ \\
\cline { 2 - 4 } & $\mathrm{PP}$ & 764 & $0.67944 \cdot 10^{-2}$ \\
\cline { 2 - 4 } & $\mathrm{FSAC}-\mathrm{PP}$ & 65 & $0.39556 \cdot 10^{-6}$ \\
\hline \multirow{3}{*}{$2^{\text {nd_Order }}$} & $\mathrm{AC}$ & 8755 & $0.16735 \cdot 10^{-3}$ \\
\cline { 2 - 4 } & $\mathrm{PP}$ & 854 & $0.99766 \cdot 10^{-2}$ \\
\cline { 2 - 4 } & $\mathrm{FSAC}-\mathrm{PP}$ & 66 & $0.20061 \cdot 10^{-6}$ \\
\hline \multirow{3}{*}{$3^{\text {rd }}$-Order } & $\mathrm{AC}$ & 9913 & $0.93892 \cdot 10^{-4}$ \\
\cline { 2 - 4 } & $\mathrm{PP}$ & 1161 & $0.33399 \cdot 10^{-2}$ \\
\cline { 2 - 4 } & $\mathrm{FSAC}-\mathrm{PP}$ & 65 & $0.39317 \cdot 10^{-6}$ \\
\hline
\end{tabular}


Table 7: Multigrid iterations and maximum of Runge-Kutta residuals at $R e=0.01$.

\begin{tabular}{||c|c|c|c||}
\hline \multicolumn{4}{|c||}{ Reynolds Number $(R e=0.01)$} \\
\hline Intercell Flux Interpolations & Solution Methods & $N_{m g}$ & $R K_{\text {maxres }}$ \\
\hline \multirow{3}{*}{$1^{\text {st }}$-Order } & $\mathrm{AC}$ & 912 & 0.25786 \\
\cline { 2 - 4 } & $\mathrm{PP}$ & 764 & $0.67973 \cdot 10^{-1}$ \\
\cline { 2 - 4 } & $\mathrm{FSAC}-\mathrm{PP}$ & 63 & $0.18301 \cdot 10^{-8}$ \\
\hline \multirow{3}{*}{$2^{\text {nd_-Order }}$} & $\mathrm{AC}$ & 1241 & 0.19437 \\
\cline { 2 - 4 } & $\mathrm{PP}$ & 854 & $0.99824 \cdot 10^{-1}$ \\
\cline { 2 - 4 } & $\mathrm{FSAC}-\mathrm{PP}$ & 63 & $0.19609 \cdot 10^{-8}$ \\
\hline \multirow{3}{*}{$3^{\text {rd_-Order }}$} & $\mathrm{AC}$ & 1230 & 0.19621 \\
\cline { 2 - 4 } & $\mathrm{PP}$ & 1161 & $0.33418 \cdot 10^{-1}$ \\
\cline { 2 - 4 } & $\mathrm{FSAC}-\mathrm{PP}$ & 62 & $0.19609 \cdot 10^{-8}$ \\
\cline { 2 - 4 }
\end{tabular}

Table 8: Multigrid iterations and maximum of Runge-Kutta residuals at $R e=0.001$.

\begin{tabular}{|c|c|c|c|}
\hline \multicolumn{4}{|c|}{ Reynolds Number $(R e=0.001)$} \\
\hline Intercell Flux Interpolations & Solution Methods & $N_{m g}$ & $R K_{\text {maxres }}$ \\
\hline \multirow{3}{*}{$1^{\text {st }}$-Order } & $\mathrm{AC}$ & 1487 & 7.69911 \\
\hline & $\mathrm{PP}$ & 764 & 0.67976 \\
\hline & FSAC-PP & 147 & $0.13595 \cdot 10^{-6}$ \\
\hline \multirow{3}{*}{$2^{n d}-$ Order } & $\mathrm{AC}$ & 1481 & 6.52887 \\
\hline & $\mathrm{PP}$ & 854 & 0.99830 \\
\hline & FSAC-PP & 84 & $0.17256 \cdot 10^{-6}$ \\
\hline \multirow{3}{*}{$3^{r d}$-Order } & $\mathrm{AC}$ & 1470 & 6.54560 \\
\hline & $\mathrm{PP}$ & 1161 & 0.33420 \\
\hline & FSAC-PP & 77 & $0.16733 \cdot 10^{-6}$ \\
\hline
\end{tabular}

Table 9: Multigrid iterations and maximum of Runge-Kutta residuals at $R e=0.0001$.

\begin{tabular}{||c|c|c|c||}
\hline \multicolumn{4}{|c|}{ Reynolds Number $(R e=0.0001)$} \\
\hline Intercell Flux Interpolations & Solution Methods & $N_{m g}$ & $R K_{\text {maxres }}$ \\
\hline \multirow{3}{*}{$1^{\text {st } \text {-Order }}$} & $\mathrm{AC}$ & 1172 & $0.16354 \cdot 10^{3}$ \\
\cline { 2 - 4 } & $\mathrm{PP}$ & 764 & 6.79768 \\
\cline { 2 - 4 } & $\mathrm{FSAC}-\mathrm{PP}$ & 81 & $0.11713 \cdot 10^{-4}$ \\
\hline \multirow{3}{*}{$2^{\text {nd_-Order }}$} & $\mathrm{AC}$ & 1169 & $0.20113 \cdot 10^{3}$ \\
\cline { 2 - 4 } & $\mathrm{PP}$ & 854 & 9.98313 \\
\cline { 2 - 4 } & $\mathrm{FSAC}-\mathrm{PP}$ & 76 & $0.13386 \cdot 10^{-4}$ \\
\hline \multirow{3}{*}{$3^{\text {rd_Order }}$} & $\mathrm{AC}$ & 1165 & $0.20293 \cdot 10^{3}$ \\
\cline { 2 - 4 } & $\mathrm{PP}$ & 1161 & 3.34202 \\
\cline { 2 - 4 } & $\mathrm{FSAC}-\mathrm{PP}$ & 123 & $0.15059 \cdot 10^{-4}$ \\
\cline { 2 - 4 } & \multicolumn{2}{|c||}{}
\end{tabular}


The relative errors of the outlet velocity component $U$ compared to the analytical solution were computed from $R e=10$ up to $10^{-4}$. On the finest grid level G4, the FSAC-PP method exhibited agreement in $\sim 0.17535 \%$ maximum relative error up to $R e=1$ while the AC and PP methods exhibited $\sim 0.50831 \%$ and $\sim 1.25491 \%$ maximum relative errors, respectively. By decreasing the Reynolds number, the maximum relative error of the AC, PP and FSAC-PP methods compared to the analytical solution was $\sim 4.19308 \%, \sim 0.00563 \%$ and $\sim 0.14231 \%$ on the fine grid at $R e=0.1$, respectively. The AC method showed good agreement with the analytically computed outlet velocity profiles up to $R e=0.1$ on each grid level; unlike the PP and the FSAC-PP methods which exhibited agreement with the analytical solution up to $R e=0.0001$. Since the lowest maximum Runge-Kutta residual of the AC method was between 0.19437 and $0.20293 \cdot 10^{3}$ when the Reynolds number was smaller than 0.1 (see Tables 7-9), thus the numerical solution was not able to resolve the fully developed laminar velocity profile at the outlet section. These results can be seen in Figs. $4-6$ at $R e=0.01,0.001$, and 0.0001 . The velocity values computed by the AC method were almost equal to zero, which means they were nearly equal to the initial condition of the velocity field. Despite of this fact, the PP and FSAC-PP methods showed fairly good agreement with the analytical solution at $R e=0.01,0.001$, and 0.0001 .

For $R e<0.1$, the AC method failed to give physically reasonable results (see Figs. 36). The results overall showed that the FSAC-PP method was more efficient than the AC and PP methods for the two-dimensional microfluidic benchmark channel. In this case, it can also be concluded that both PP and FSAC-PP methods were capable of handling extremely low Reynolds numbers more accurately than the AC method. The computational experience gained in this study also showed that the FSAC-PP method performed at a very slow convergence rate when the Reynolds number was smaller than $10^{-4}$. This result suggests that the FSAC-PP method is valid for Reynolds numbers up to $10^{-4}$. Consequently, further investigation is required to understand the convergence properties of the FSAC-PP method for $\operatorname{Re}<10^{-4}$; and this approach should be further tested for complex microfluidic problems when a small Reynolds number flow occurs.

\subsection{Two-dimensional steady-state flow in a lid driven square cavity at moderate Reynolds numbers ( $R e=100,400$, and 1000)}

In this subsection, we choose the two-dimensional, steady-state, incompressible flow problem in a lid driven square cavity as a benchmark problem to validate the proposed FSAC-PP method at moderate Reynolds numbers. This is one of the most frequently used benchmark test cases in the field of CFD for validation purpose, because reliable numerical data are available in the literature. For the dimensionless velocity components $V$ and $U$ along the horizontal and vertical centre-lines, the results are compared to the AC and PP methods, and the classical numerical data of Ghia et al. [52] at $R e=100,400$, and 1000. For this study, 45 simulations were performed by using three different methods with five different intercell flux interpolation schemes (from first- up to ninth-order) at three different Reynolds numbers. The incompressible Navier-Stokes equations were 


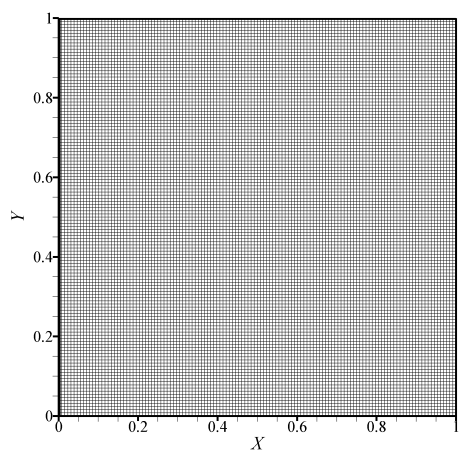

Figure 8: Computational domain and equidistant grid for a square cavity by using dimensionless spatial coordinates.

solved by applying a pseudo-time stepping procedure until the steady-state solution is achieved. The domain was a unit square (see Fig. 8). For these Reynolds numbers, the numerical grid consists of $129 \times 129$ node points $128 \times 128$ control cells on the fine grid, which was the same grid as used by Ghia et al. [52], thus a grid convergence study was not performed for this benchmark problem. For the simulations, the AC parameter $\beta$ was chosen to be equal to 10, and the $C F L_{\text {inv }}$ and $C F L_{v i s}$ numbers are equal to 0.5 .

A mathematical task can be formulated in terms of boundary and initial conditions for the two-dimensional laminar flow in a lid driven square cavity. For this benchmark problem, dimensionless quantities were used, thus the spatial coordinates were normalized by the characteristic length $l$ of the square cavity. The dimensional velocity components $u$ and $v$ were normalized by the average velocity $\tilde{u}$ of the moving lid on the top. No-slip boundary conditions were prescribed for the dimensionless velocity components $U$ and $V$ on the non-moving side walls and the bottom wall as $U_{\text {wall }}=V_{\text {wall }}=0$. Dirichlet-type boundary conditions were imposed for the velocity components $U$ and $V$ on the moving top wall as $U_{l i d}=1$ and $V_{l i d}=0$. It is essential to obtain a unique solution for the incompressible Navier-Stokes equations for this benchmark problem, thus the reference static pressure was taken to be equal to zero in the lower left corner of the square cavity. Therefore Neumann-type boundary conditions can be prescribed for the pressure on the non-moving and moving walls as $\partial P / \partial n=0$, because the pressure was determined up to a constant value to avoid infinite particular solutions of the fully Neumann-type boundary value problem. The Neumann-type boundary conditions can be considered mathematically correct for the pressure (see [47]). The initial conditions for the velocity components $U, V$ and the pressure values $P$ were equal to zero in each interior cell.

The dimensionless velocity profiles, including the streamline patterns, compared to the numerical data of Ghia et al. [52] can be seen in Figs. 10-15. First of all, the results of the low-order (first-order) interpolation scheme were investigated at $R e=100$ (see Figs. 10-15). For the velocity component $V$ along the horizontal centre-line, the maximum relative errors of the AC, PP and FSAC-PP methods were $\sim 0.19925, \sim 0.42650$ and 
$\sim 0.07362$, respectively (see Table 10). For the velocity component $U$ along the vertical centre-line, the maximum relative errors of the AC, PP and FSAC-PP methods were $\sim 15.67207, \sim 13.76639$ and $\sim 0.70087$, respectively (see Table 11). In terms of accuracy, these results showed that the FSAC-PP method gave the most accurate agreement with the numerical data of Ghia et al. [52] for both velocity components $V$ and $U$ by using first-order interpolation scheme at $R e=100$ (see Figs. 10 and 15).

It was also observed that the primary large eddy and the corner vortices, compared to the streamline patterns in [52], were captured correctly by employing the FSAC-PP method with a first-order scheme (see Fig. 15). It is essential to note that neither the velocity profiles nor the corner vortices were resolved by the AC and PP methods by applying a first-order interpolation scheme (see Figs. 10 and 15).

For $R e=100$, the convergence history of the pseudo-time stepping procedure is shown in Fig. 16, which can be characterized by the maximum value of the Runge-Kutta residuals versus the total number of multigrid iterations on the fine grid until the steady-state solution is achieved. The total number of multigrid iterations of the AC, PP and FSAC-PP methods were 107, 7280 and 97, respectively (see Fig. 16 and Table 13). With respect to numerical efficiency, the FSAC-PP method required the lowest number of multigrid cycles of 97, compared to the AC and PP methods. Due to the small time-step, the highest number of multigrid cycles of 7280 was obtained by using the PP method (see Fig. 16 and Table 13). For this benchmark, the FSAC-PP method was the most accurate and efficient method compared to the AC and PP methods when a first-order interpolation scheme was employed at each investigated Reynolds number (see Figs. 10 and 15).

For $R e=100$, when second-order interpolation was employed, the maximum relative errors of the AC, PP and FSAC-PP methods for the velocity component $V$ were $\sim 0.05110$, $\sim 0.27482$ and $\sim 0.05531$, respectively (see Table 10 ). For the velocity component $U$, the maximum relative errors of these methods were $\sim 0.27663, \sim 0.63502$ and $\sim 0.08482$, respectively (see Table 11). For velocity components $V$ and $U$ in this case, the AC and FSAC-PP methods were more accurate than the PP method. Unlike when the first-order scheme was employed, the FSAC-PP method was, overall, more accurate than the AC and PP methods for this benchmark problem (see Fig. 10). By increasing the order of the interpolation scheme for the convective terms, when third-order interpolation was employed, the maximum relative errors of the AC, PP and FSAC-PP methods for the velocity component $V$ were $\sim 0.05420, \sim 0.07968$ and $\sim 0.05270$, respectively (see Table 10). For the velocity component $U$, the maximum relative errors of these methods were $\sim 0.23427, \sim 0.22983$ and $\sim 0.11597$, respectively (see Table 11). It can also be seen from the results of this case that the accuracy of the AC and PP methods were similar to each other for the velocity component $V$, and the proposed FSAC-PP method was the most accurate for the velocity component $U$. In terms of efficiency, the results showed again that the unified FSAC-PP method required the lowest number of multigrid iterations $N_{m g}$ on the fine grid compared to the AC and PP methods at $R e=100$ (see Fig. 16 and Table 13).

The maximum relative errors of the fifth- and ninth-order Weighted Essentially NonOscillatory (WENO) schemes have been summarized in Tables 10 and 11. The numerical 


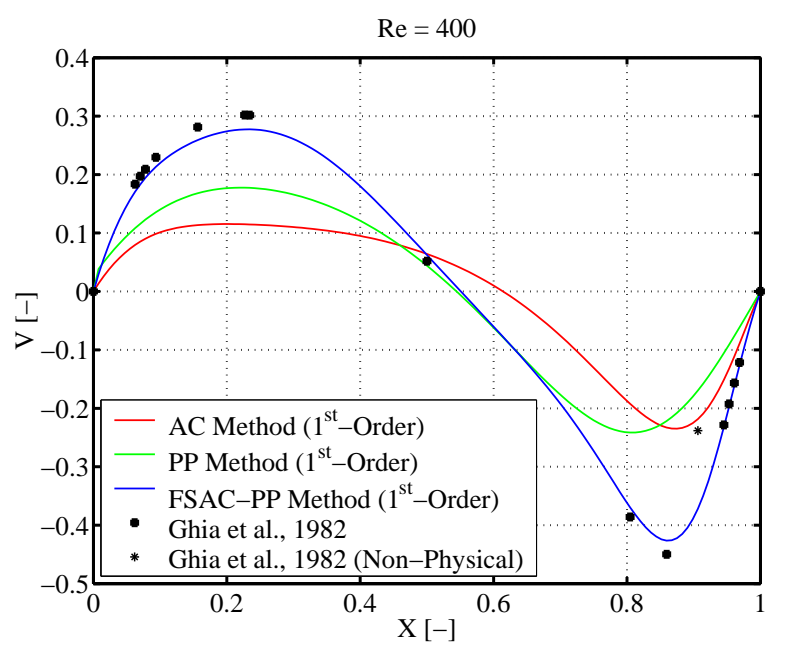

Figure 9: Computational results of the AC, PP, and FSAC-PP methods for dimensionless velocity component $V$ along the horizontal centre-line of the square cavity using a $1^{\text {st }}$-order interpolation scheme compared to the data of Ghia et al. [52] at $\operatorname{Re}=400$.

results of high-order schemes suggested, similarly to the use of third-order interpolation, that the accuracy of the AC and FSAC-PP methods were similar to each other for the velocity component $V$, and again the unified FSAC-PP method was the most accurate for the horizontal velocity component $U$. This also means that the numerically computed velocity profiles in the centre-lines did not change significantly by employing higher than a third-order interpolation for this benchmark problem. The reason is that the grid resolution of $129 \times 129$ node points, is a fine mesh for this test case, thus the high-order schemes did not improve further the accuracy of the solution (see Figs. 12-14).

The results of the AC and PP methods obtained by the first-order scheme for the convective terms led to non-physical solutions at $R e=400$ and 1000, respectively even if the solutions were converged to the threshold value of $10^{-9}$ (see Figs. 10, 15 and 16). Note that the numerical data of Ghia et al. [52, p. 399, Table II] was also non-physical at the grid point number $117(X=0.9063, V=-0.23827)$, which is shown in Fig. 9 depicted by '*'. It is important to emphasize that the non-physical data were excluded from the computation of the maximum relative errors, which are summarized in Tables 10 and 11.

For $R e=400$, the results showed that when the second-order interpolation was used for the velocity components $V$ and $U$ (see Tables 10-11), the PP method was more accurate than the AC and FSAC-PP methods for the velocity component $V$, and again the FSACPP method was the most accurate for the velocity component $U$ (see Fig. 11). This result was also valid for the velocity component $V$ by employing a third-order interpolation (see Fig. 12 and Table 10). Unlike when the second-order scheme was employed, the AC method exhibited the lowest maximum relative error for the velocity component $U$ (see Table 11). In the cases of fifth- and ninth-order WENO schemes, the PP method was more accurate than the AC and FSAC-PP methods for the velocity component $V$, and 
Table 10: Maximum relative errors of the dimensionless velocity component $V$ compared to the numerical data of Ghia et al. [52] at $R e=100,400$, and 1000, respectively.

\begin{tabular}{||c|c|c|c|c||}
\hline \multicolumn{5}{|c|}{ Maximum Relative Errors along Horizontal Centre-Line [-] } \\
\hline Intercell Flux Interpolations & Solution Methods & $R e=100$ & $R e=400$ & $R e=1000$ \\
\hline \multirow{3}{*}{$1^{\text {st }- \text { Order }}$} & $\mathrm{AC}$ & 0.19925 & 0.61997 & 1.15918 \\
\cline { 2 - 5 } & $\mathrm{PP}$ & 0.42650 & 0.53882 & 0.82317 \\
\cline { 2 - 5 } & $\mathrm{FSAC-PP}$ & 0.07362 & 0.56081 & 0.28518 \\
\hline \multirow{3}{*}{$2^{\text {nd }}$-Order } & $\mathrm{AC}$ & 0.05110 & 0.61561 & 0.10497 \\
\cline { 2 - 5 } & $\mathrm{PP}$ & 0.27482 & 0.58735 & 0.45352 \\
\cline { 2 - 5 } & $\mathrm{FSAC-PP}$ & 0.05531 & 0.61311 & 0.57405 \\
\hline \multirow{3}{*}{$3^{\text {rd }}$-Order } & $\mathrm{AC}$ & 0.05420 & 0.62387 & 0.04062 \\
\cline { 2 - 5 } & $\mathrm{PP}$ & 0.07968 & 0.53539 & 0.43522 \\
\cline { 2 - 5 } & $\mathrm{FSAC-PP}$ & 0.05270 & 0.61038 & 0.02942 \\
\hline \multirow{3}{*}{$5^{\text {th }}$-WENO } & $\mathrm{AC}$ & 0.05415 & 0.62492 & 0.03863 \\
\cline { 2 - 5 } & $\mathrm{PP}$ & 0.07575 & 0.50669 & 0.45148 \\
\cline { 2 - 5 } & $\mathrm{FSAC-PP}$ & 0.05283 & 0.60976 & 0.10794 \\
\hline \multirow{3}{*}{$9^{t h}$-WENO } & $\mathrm{AC}$ & 0.05391 & 0.62612 & 0.04084 \\
\cline { 2 - 5 } & $\mathrm{PP}$ & 0.07455 & 0.53552 & 0.47184 \\
\cline { 2 - 5 } & $\mathrm{FSAC-PP}$ & 0.05277 & 0.61141 & 0.05262 \\
\hline
\end{tabular}

Table 11: Maximum relative errors of the dimensionless velocity component $U$ compared to the numerical data of Ghia et al. [52] at $R e=100,400$, and 1000 , respectively.

\begin{tabular}{||c|c|c|c|c||}
\hline \multicolumn{5}{|c|}{ Maximum Relative Errors along Vertical Centre-Line [-] } \\
\hline Intercell Flux Interpolations & Solution Methods & $R e=100$ & $R e=400$ & $R e=1000$ \\
\hline \multirow{3}{*}{$1^{\text {st }}$ _Order } & $\mathrm{AC}$ & 15.67207 & 3.11797 & 1.16409 \\
\cline { 2 - 5 } & $\mathrm{PP}$ & 13.76639 & 8.12039 & 3.42777 \\
\cline { 2 - 5 } & $\mathrm{FSAC}-\mathrm{PP}$ & 0.70087 & 0.19398 & 0.13116 \\
\hline \multirow{3}{*}{$2^{\text {nd }}$ _Order } & $\mathrm{AC}$ & 0.27663 & 0.05174 & 0.12761 \\
\cline { 2 - 5 } & $\mathrm{PP}$ & 0.63502 & 0.21816 & 0.31473 \\
\cline { 2 - 5 } & $\mathrm{FSAC}-\mathrm{PP}$ & 0.08482 & 0.03716 & 0.44063 \\
\hline \multirow{3}{*}{$3^{\text {rd }}$-Order } & $\mathrm{AC}$ & 0.23427 & 0.03705 & 0.02469 \\
\cline { 2 - 5 } & $\mathrm{PP}$ & 0.22983 & 0.46706 & 0.26077 \\
\cline { 2 - 5 } & $\mathrm{FSAC}-\mathrm{PP}$ & 0.11597 & 0.05844 & 0.06324 \\
\hline \multirow{3}{*}{$5^{\text {th }}$-WENO } & $\mathrm{AC}$ & 0.24897 & 0.03432 & 0.02355 \\
\cline { 2 - 5 } & $\mathrm{PP}$ & 0.29880 & 0.58807 & 0.39702 \\
\cline { 2 - 5 } & $\mathrm{FSAC}-\mathrm{PP}$ & 0.12395 & 0.06039 & 0.11584 \\
\hline \multirow{3}{*}{$9^{\text {th }}$-WENO } & $\mathrm{AC}$ & 0.24727 & 0.03205 & 0.02236 \\
\cline { 2 - 5 } & $\mathrm{PP}$ & 0.36641 & 0.46733 & 0.28609 \\
\cline { 2 - 5 } & $\mathrm{FSAC}-\mathrm{PP}$ & 0.12054 & 0.05240 & 0.07939 \\
\hline
\end{tabular}


(a) $\operatorname{Re}=100$

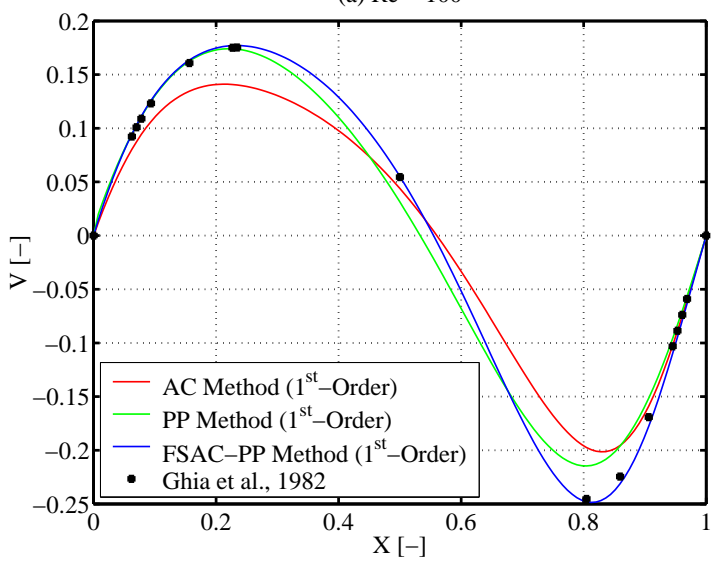

(c) $\operatorname{Re}=400$

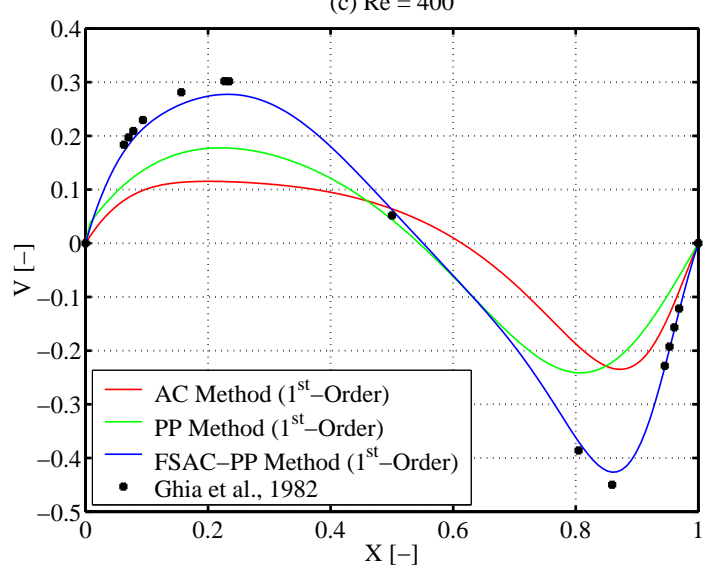

(e) $\operatorname{Re}=1000$

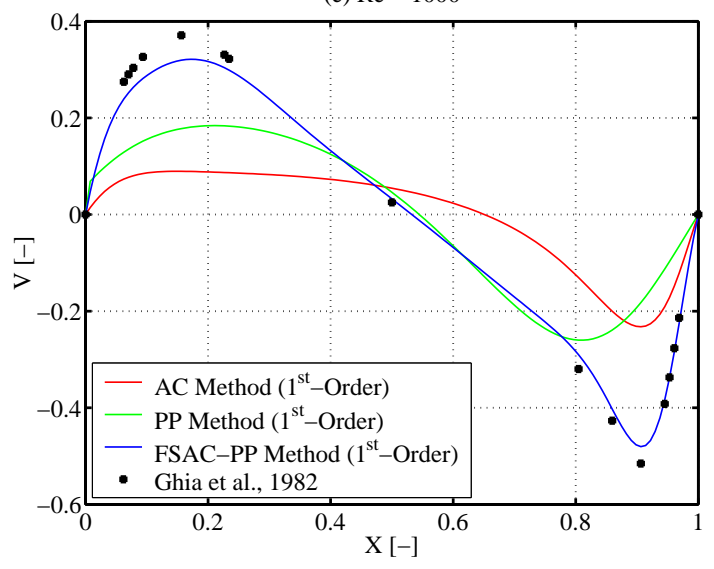

(b) $\operatorname{Re}=100$

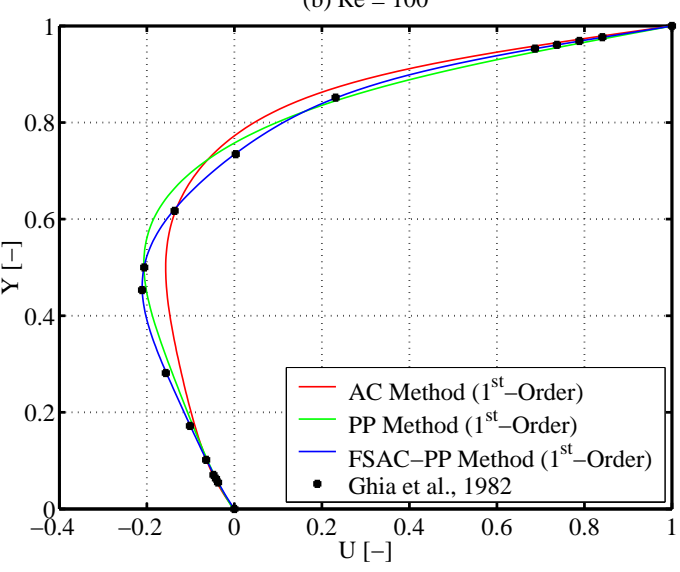

(d) $\mathrm{Re}=400$

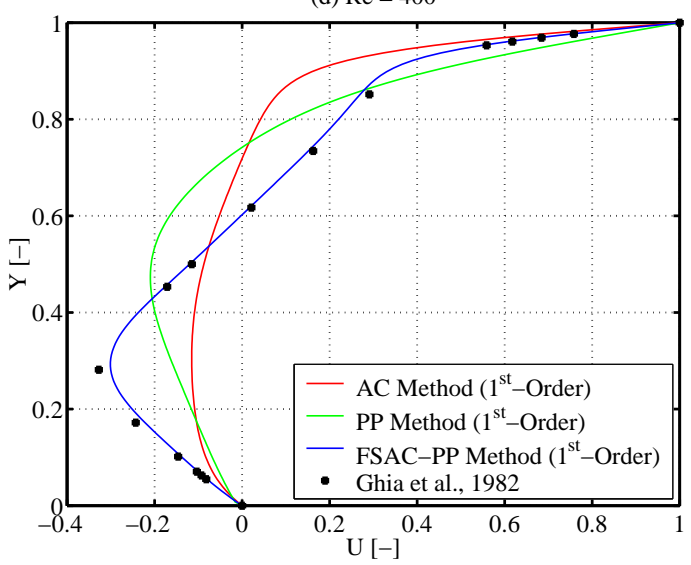

(f) $\operatorname{Re}=1000$

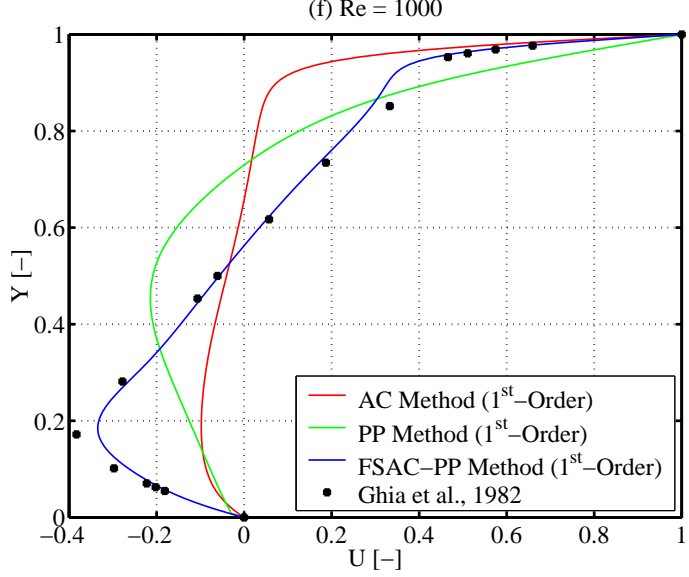

Figure 10: Computational results of the AC, PP, and FSAC-PP methods for dimensionless velocity components $V$ and $U$ along (a), (c), (e) horizontal and (b), (d), (f) vertical centre-lines of the square cavity using a $1^{\text {st }}$-order interpolation scheme compared to the numerical results of Ghia et al. [52] at $R e=100,400,1000$, respectively. 
(a) $\operatorname{Re}=100$

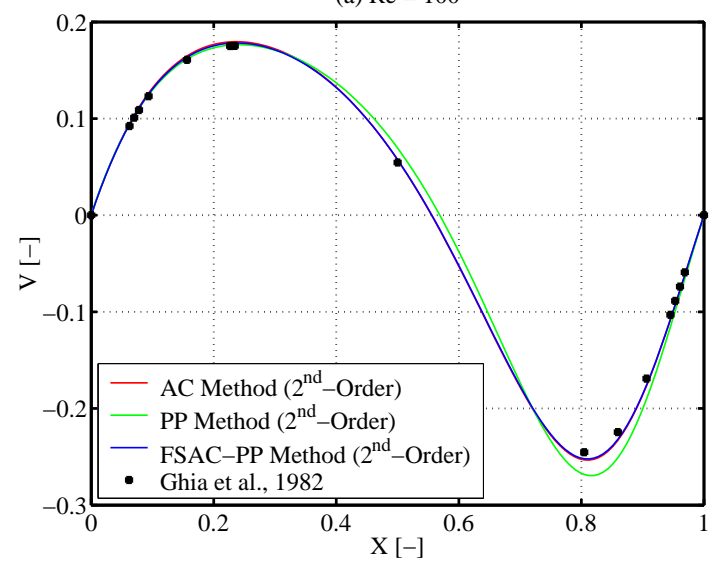

(c) $\operatorname{Re}=400$

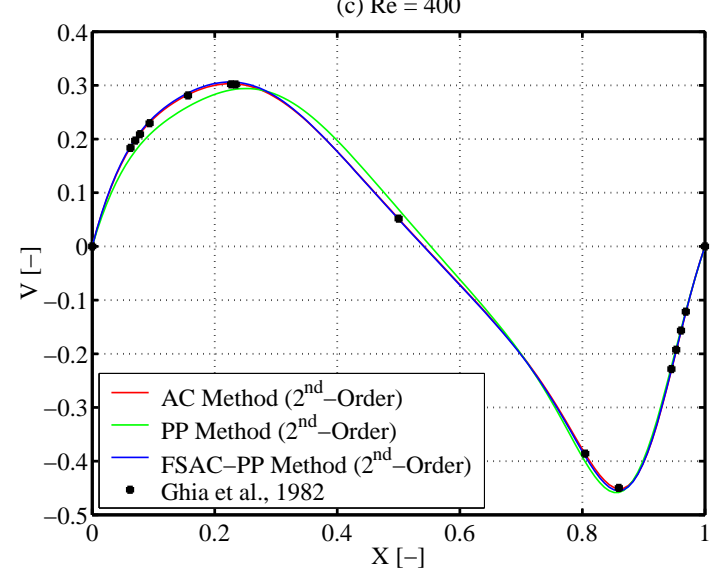

(e) $\operatorname{Re}=1000$

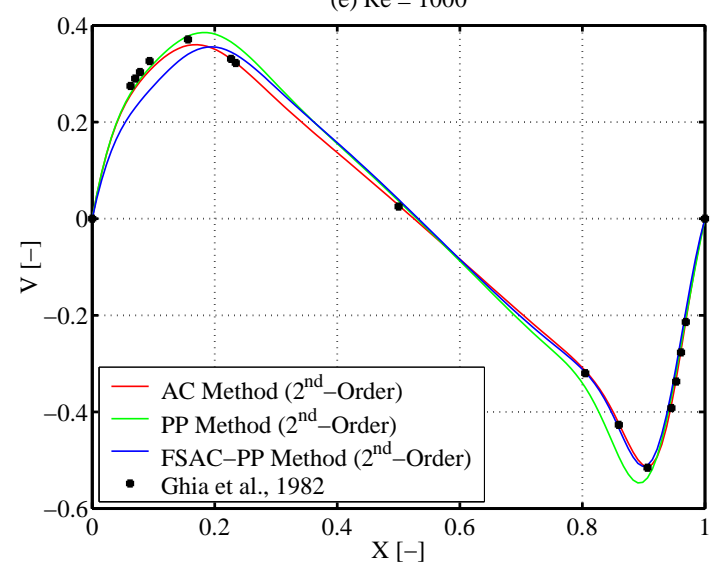

(b) $\operatorname{Re}=100$

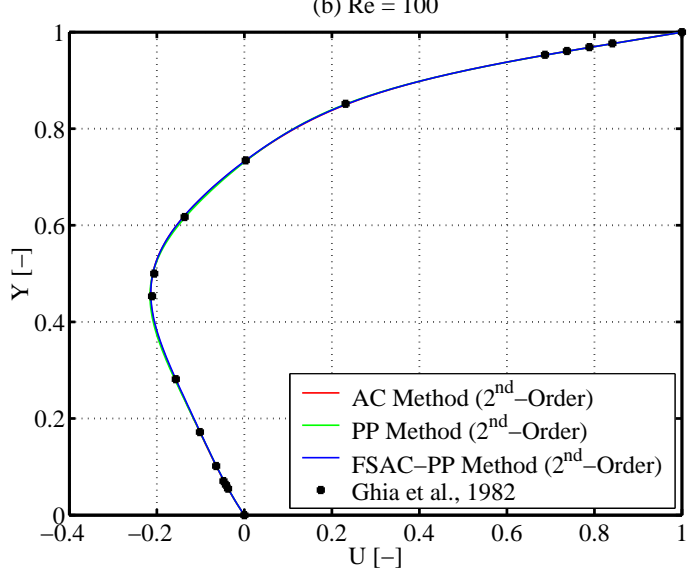

(d) $\operatorname{Re}=400$

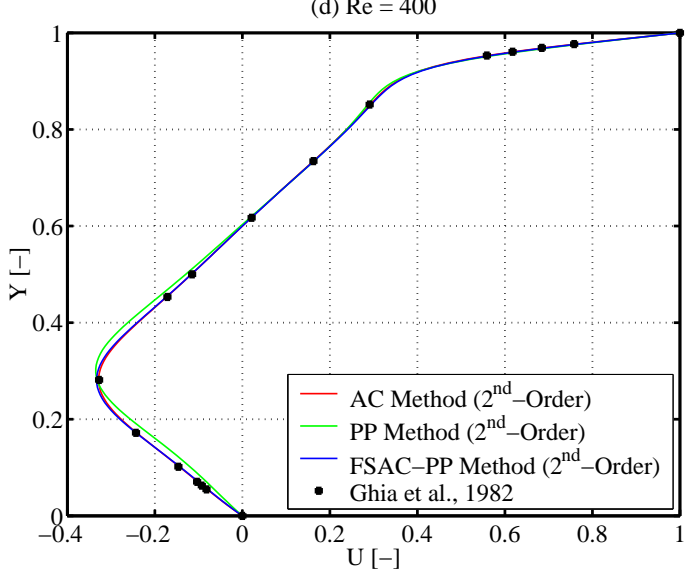

(f) $\operatorname{Re}=1000$

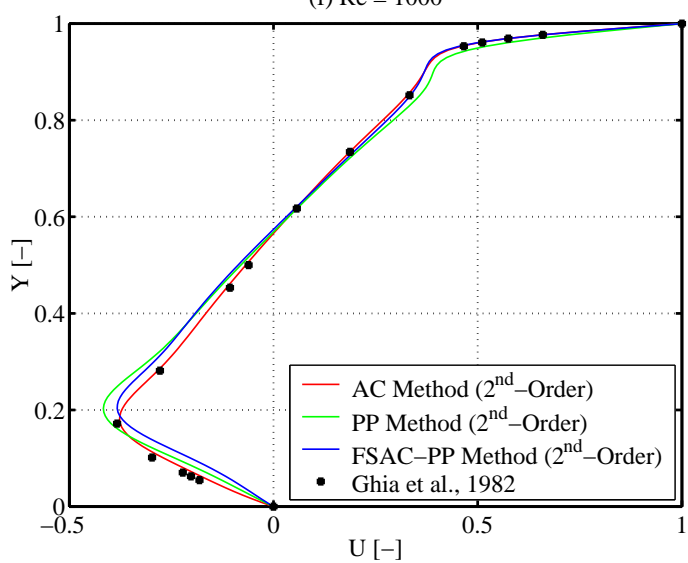

Figure 11: Computational results of the AC, PP, and FSAC-PP methods for dimensionless velocity components $V$ and $U$ along (a), (c), (e) horizontal and (b), (d), (f) vertical centre-lines of the square cavity using a $2^{n d}$-order interpolation scheme compared to the numerical results of Ghia et al. [52] at $R e=100,400,1000$, respectively. 
(a) $\operatorname{Re}=100$

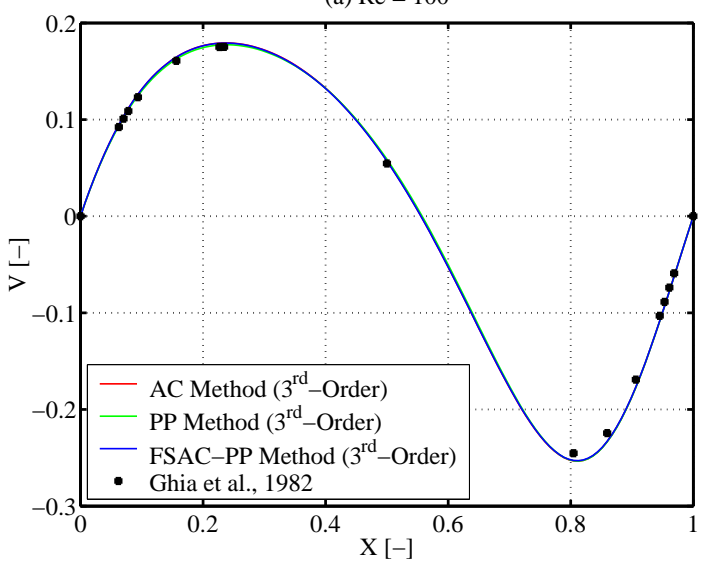

(c) $\operatorname{Re}=400$

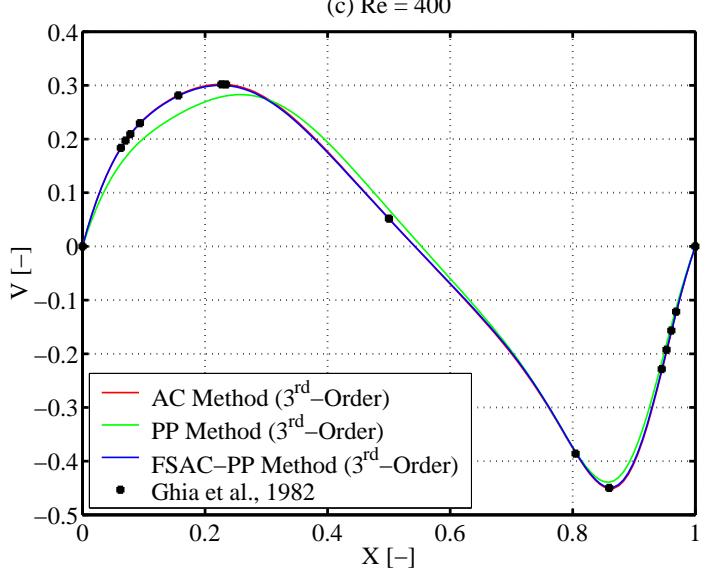

(e) $\operatorname{Re}=1000$

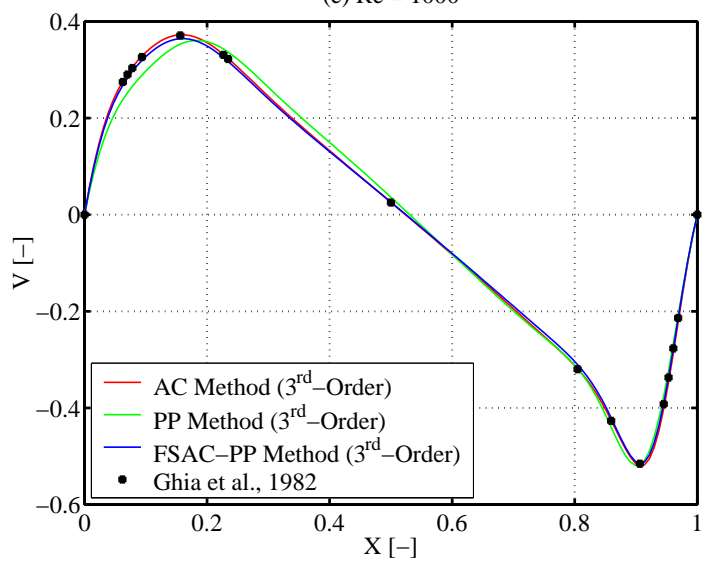

(b) $\mathrm{Re}=100$

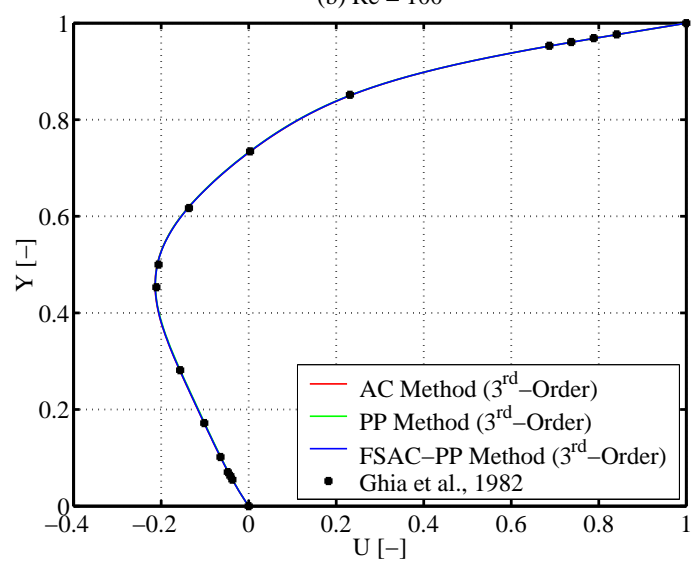

(d) $\operatorname{Re}=400$

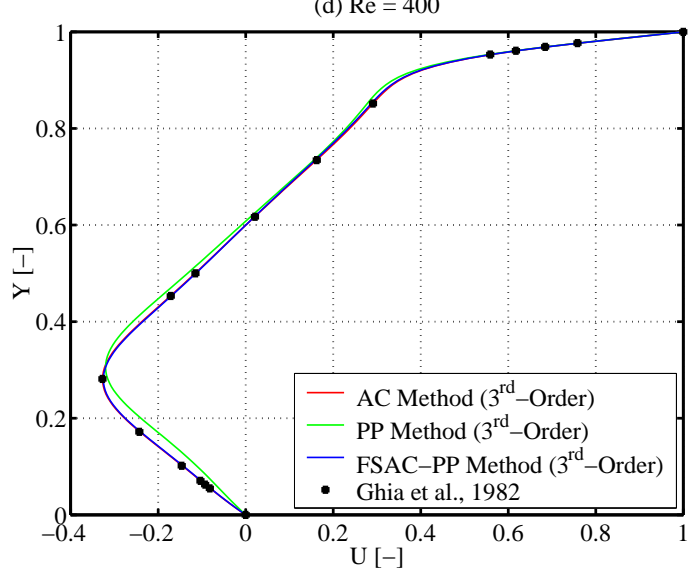

(f) $\operatorname{Re}=1000$

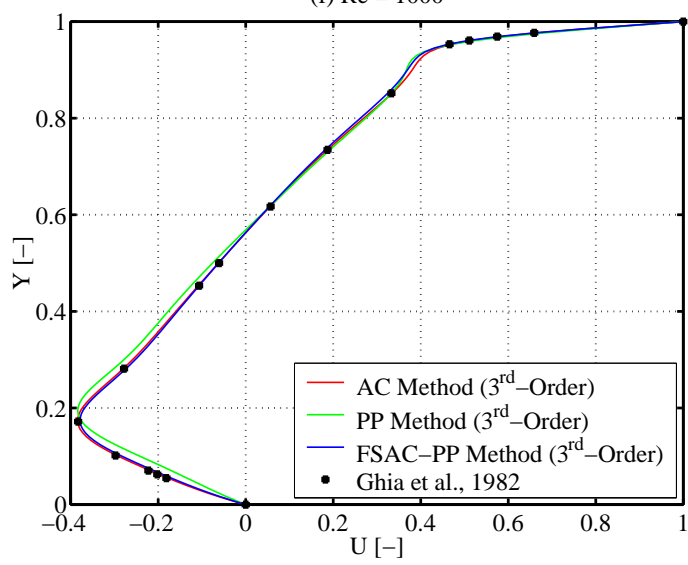

Figure 12: Computational results of the AC, PP, and FSAC-PP methods for dimensionless velocity components $V$ and $U$ along (a), (c), (e) horizontal and (b), (d), (f) vertical centre-lines of the square cavity using a $3^{r d}$-order interpolation scheme compared to the numerical results of Ghia et al. [52] at $R e=100,400,1000$, respectively. 

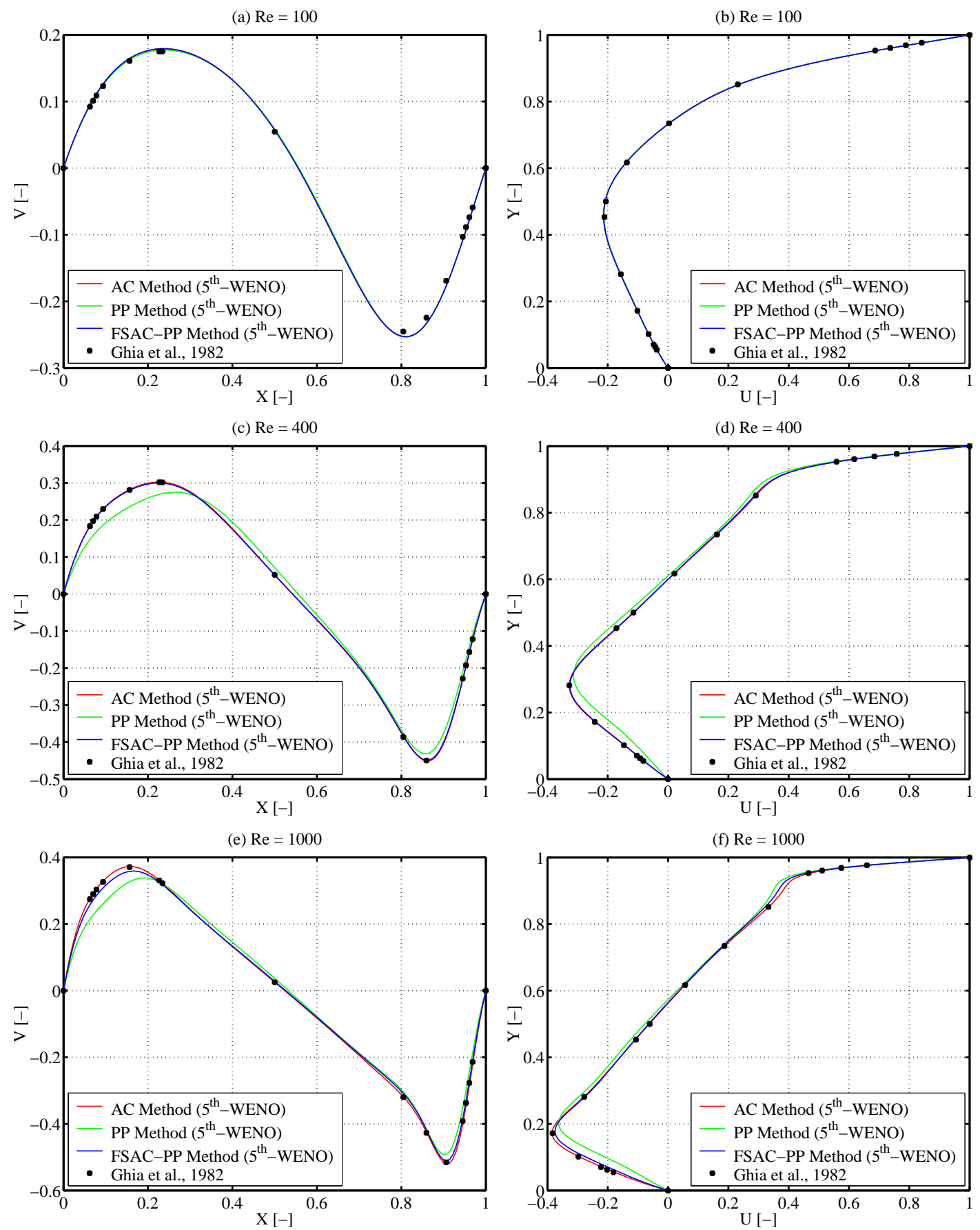

Figure 13: Computational results of the AC, PP, and FSAC-PP methods for dimensionless velocity components $V$ and $U$ along (a), (c), (e) horizontal and (b), (d), (f) vertical centre-lines of the square cavity using $5^{\text {th }}$-order WENO interpolation scheme compared to the numerical results of Ghia et al. [52] at $R e=100,400,1000$, respectively. 

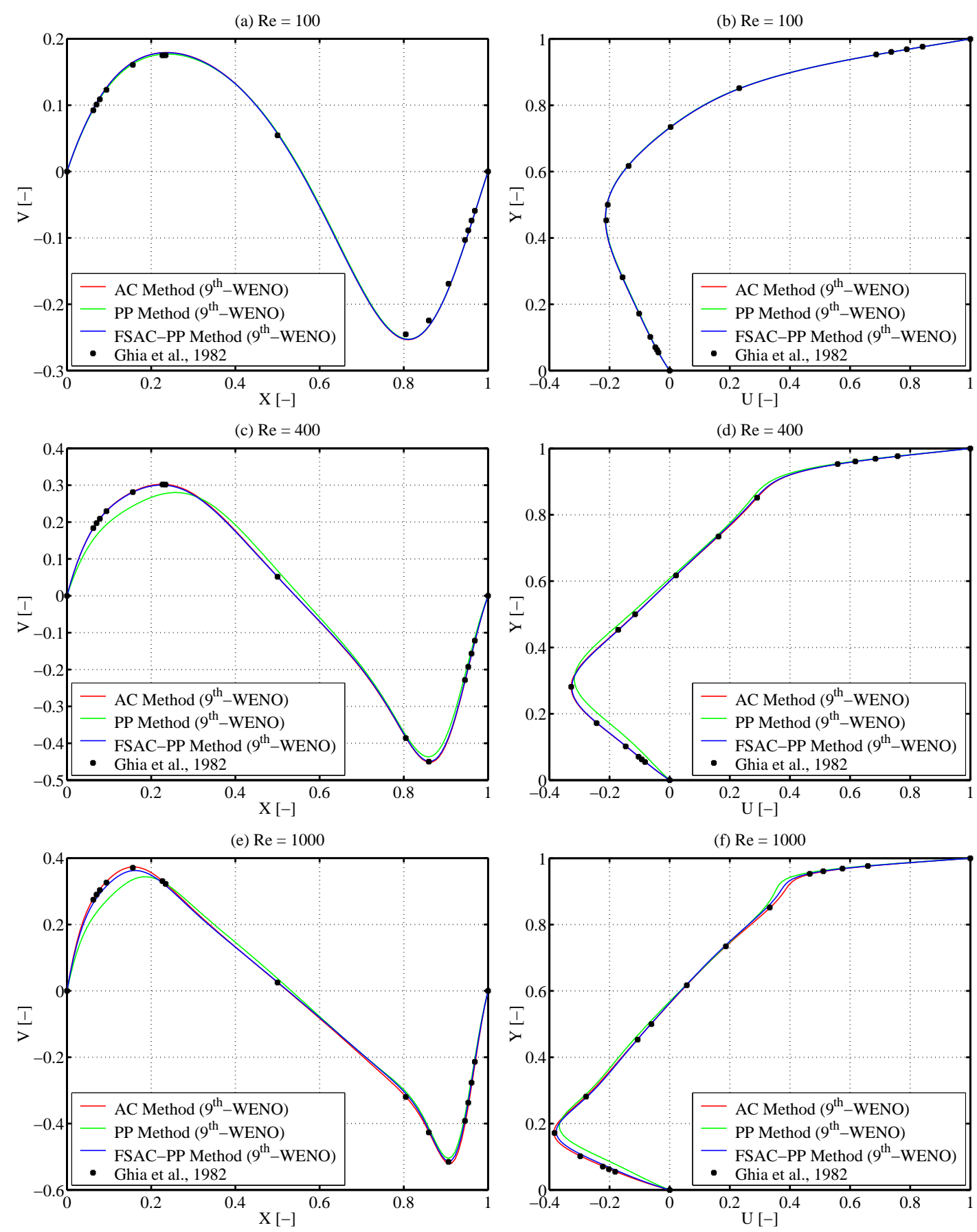

Figure 14: Computational results of the AC, PP, and FSAC-PP methods for dimensionless velocity components $V$ and $U$ along (a), (c), (e) horizontal and (b), (d), (f) vertical centre-lines of the square cavity using $9^{\text {th }}$-order WENO interpolation scheme compared to the numerical results of Ghia et al. [52] at $R e=100,400,1000$, respectively. 


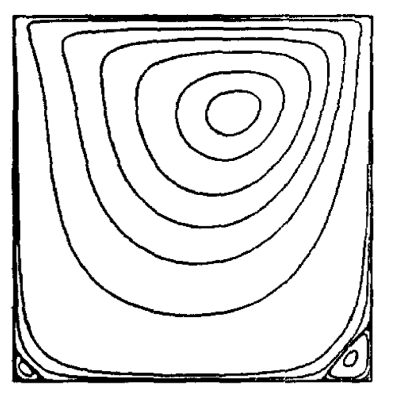

(a) $\operatorname{Re}=100$, Ghia et al. [52]

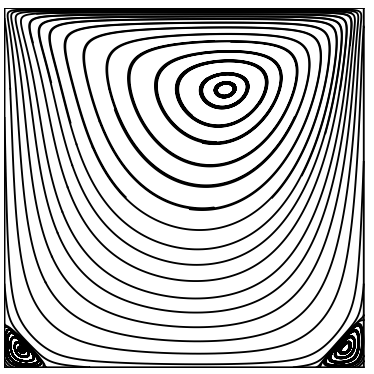

(d) $R e=100, \mathrm{AC}-1^{\text {st }}$-Order

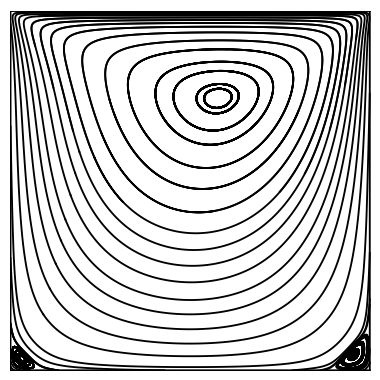

(g) $R e=100, \mathrm{PP}-1^{\text {st }}$-Order

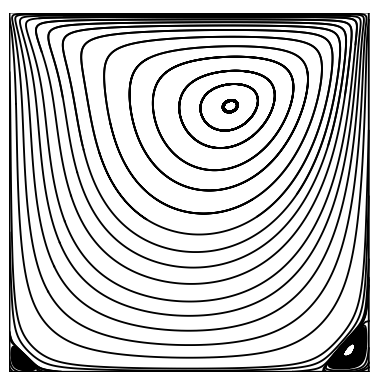

(j) $R e=100$, FSAC-PP- $1^{\text {st }}$-Order

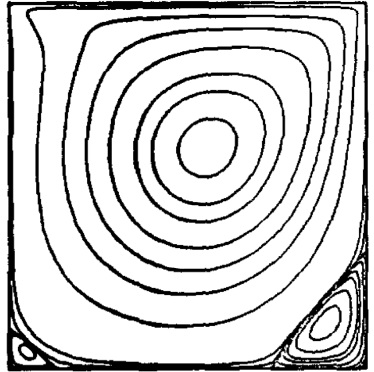

(b) $R e=400$, Ghia et al. [52]

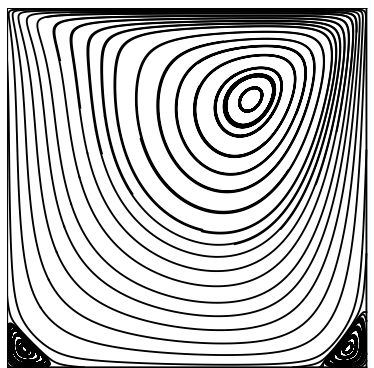

(e) $R e=400, \mathrm{AC}-1^{\text {st }}$-Order

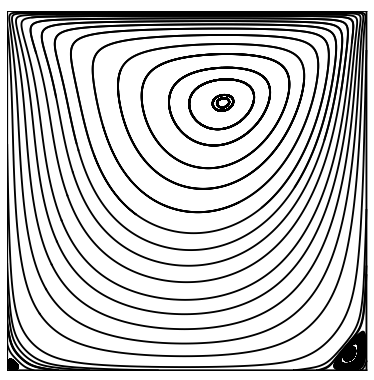

(h) $R e=400, \mathrm{PP}-1^{\text {st }}$-Order

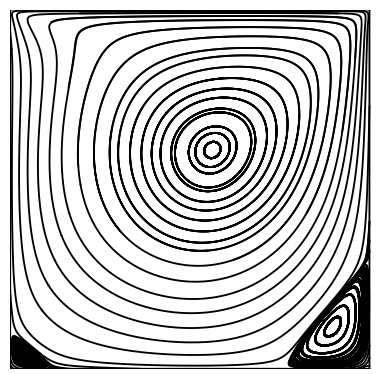

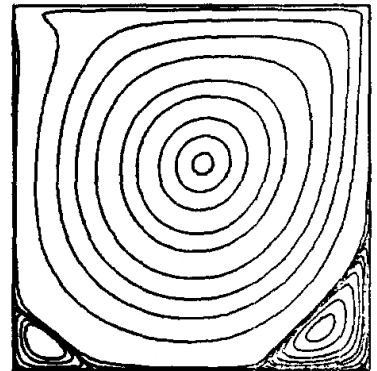

(c) $\operatorname{Re}=1000$, Ghia et al. [52]

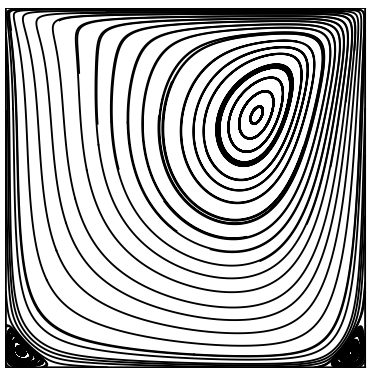

(f) $\operatorname{Re}=1000, \mathrm{AC}-1^{\text {st }}$-Order

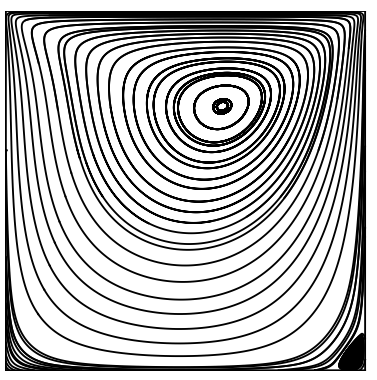

(i) $\operatorname{Re}=1000, \mathrm{PP}-1^{\text {st }}$-Order

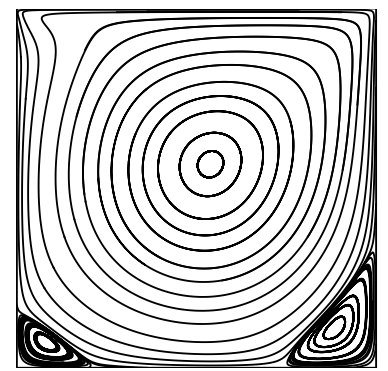

(k) $R e=400$, FSAC-PP-1 ${ }^{\text {st }}$-Order (l) $R e=1000$, FSAC-PP- $1^{\text {st }}$-Order

Figure 15: Streamline patterns of the AC, PP, and FSAC-PP methods in the square cavity using a $1^{\text {st }}$-order interpolation scheme compared to the numerical results of Ghia et al. [52] at $R e=100,400,1000$, respectively. 

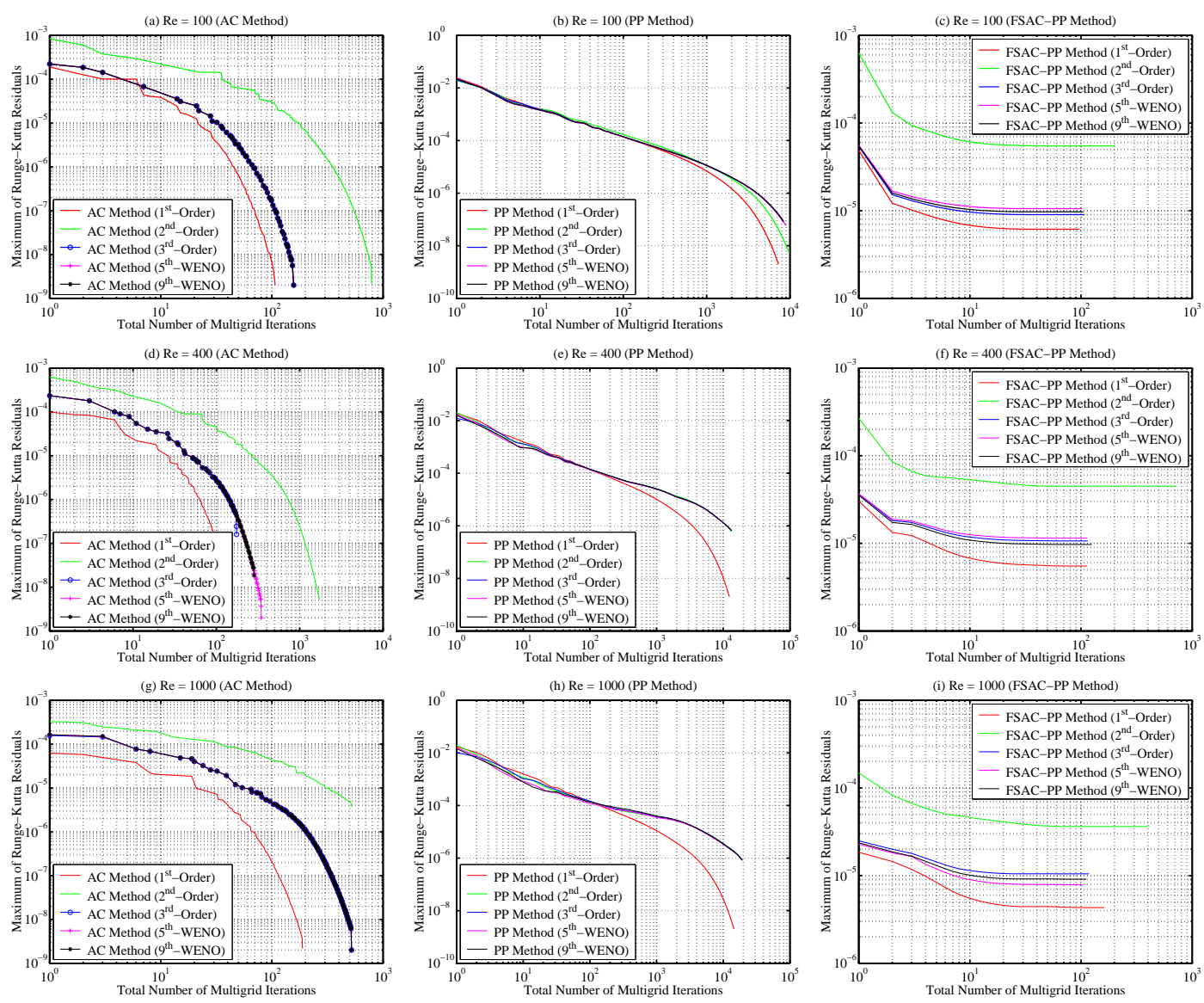

Figure 16: Comparison of the numerical convergence of the AC, PP, and FSAC-PP methods; maximum values of Runge-Kutta residuals versus total number of multigrid iterations on the fine grid at $R e=100,400$, and 1000 , respectively.

the AC and FSAC-PP methods were more accurate than the PP method for the velocity component $U$ (see Figs. 13 and 14, and Tables 10 and 11). For $R e=400$, the results of high-order schemes also did not improve further the accuracy of the solution compared with the third-order scheme (see Figs. 12, 13 and 14).

For $R e=1000$, the results again showed that the FSAC-PP method gave the most accurate agreement with the numerical data of [52] when a first-order scheme was employed (see Fig. 10, and Tables 10 and 11). The AC method was more accurate than the PP and FSAC-PP methods when fifth- and ninth-order WENO schemes were used, compared to the other schemes (see Figs. 13 and 14).

The overall results suggest that the accuracy of the AC and FSAC-PP methods were similar to each other for this benchmark problem (see Tables 10 and 11). The FSACPP approach was proven to be the most efficient method compared to the AC and PP methods at $R e=100,400$, and 1000 (see Fig. 16 and Tables 12-13). Furthermore, the 
Table 12: Computational times for a stationary flow problem in a lid driven square cavity at $\operatorname{Re}=100,400$, and 1000 on a Dual-Core E5300 $2.60 \mathrm{GHz}$ computer by employing a $3^{\text {rd }}$-order interpolation scheme for the convective term.

\begin{tabular}{||c|c|c|c||}
\hline \multirow{2}{*}{} & \multicolumn{3}{|c||}{ Reynolds Numbers (Re) } \\
\cline { 2 - 4 } & 100 & 400 & 1000 \\
\hline Solution Methods & \multicolumn{3}{|c||}{ Computational Times (Minutes) } \\
\hline AC & 89 & 71 & 244 \\
\hline PP & 129 & 251 & 321 \\
\hline FSAC-PP & 86 & 57 & 105 \\
\hline
\end{tabular}

Table 13: Total number of multigrid iterations on the fine grid using five different interpolation schemes at $R e=100,400$, and 1000, respectively.

\begin{tabular}{|c|c|c|c|c|}
\hline \multicolumn{5}{|c|}{ Total Number of Multigrid Iterations on the Fine Grid } \\
\hline Intercell Flux Interpolations & Solution Methods & $\operatorname{Re}=100$ & $\operatorname{Re}=400$ & $\operatorname{Re}=1000$ \\
\hline \multirow{3}{*}{$1^{\text {st }}$-Order } & $\mathrm{AC}$ & 107 & 159 & 189 \\
\hline & PP & 7280 & 12360 & 14490 \\
\hline & FSAC-PP & 97 & 113 & 162 \\
\hline \multirow{3}{*}{$2^{\text {nd }}$-Order } & $\mathrm{AC}$ & 788 & 1716 & 526 \\
\hline & PP & 9780 & 13180 & 19380 \\
\hline & FSAC-PP & 202 & 722 & 403 \\
\hline \multirow{3}{*}{$3^{r d}$-Order } & $\mathrm{AC}$ & 157 & 175 & 521 \\
\hline & PP & 8350 & 13180 & 19310 \\
\hline & FSAC-PP & 107 & 115 & 118 \\
\hline \multirow{3}{*}{$5^{\text {th }}-\mathrm{WENO}$} & $\mathrm{AC}$ & 157 & 346 & 521 \\
\hline & $\mathrm{PP}$ & 8940 & 12600 & 18460 \\
\hline & FSAC-PP & 102 & 114 & 104 \\
\hline \multirow{3}{*}{$9^{\text {th }}-\mathrm{WENO}$} & $\mathrm{AC}$ & 157 & 283 & 521 \\
\hline & PP & 8370 & 13400 & 18990 \\
\hline & FSAC-PP & 105 & 125 & 112 \\
\hline
\end{tabular}

proposed unified FSAC-PP approach also provided accurate and efficient solutions by employing a first-order (low-order) scheme as discussed above.

\subsection{Three-dimensional unsteady turbulent flow in a lid driven cubical cavity at a higher Reynolds number $(R e=10000)$}

The FSAC-PP method has been further validated for an unsteady, incompressible, turbulent flow and the results were compared to the AC and PP methods, and the experimental data of Prasad and Koseff [53] for a lid driven cubical cavity at $R e=10000$. This benchmark problem is of central interest in the field of CFD, because both simulations and measurements are available in the literature $[53,55,56]$. Therefore this problem was chosen to 


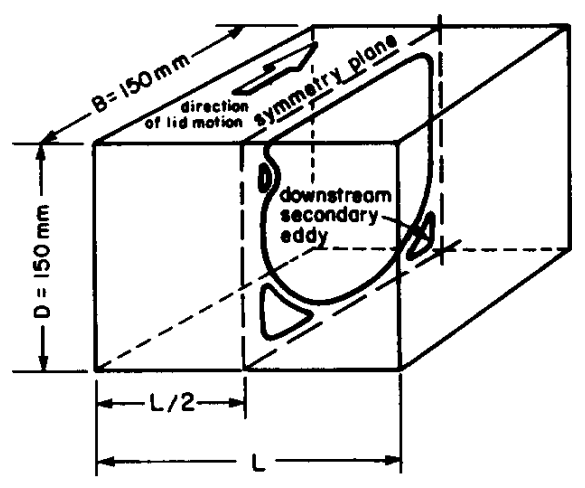

Figure 17: Geometrical dimensions of the cubical cavity configuration measured by Prasad et al. [53, p. 209] at $L=B=D=150 \mathrm{~mm}$ with spanwise aspect ratio of $1: 1$.

measure the computational time of the AC, PP, and FSAC-PP methods for an unsteady flow at a higher Reynolds number. The geometry of the full measurement configuration of Prasad and Koseff can be seen in Fig. 17 [53, p. 209]. The dimensions of the computational domain are equal to the cubical cavity of the experiment: $L=B=D=150 \mathrm{~mm}$ at a spanwise aspect ratio (SAR) of 1:1 [53] (see Fig. 18). The numerical grid consists of a $64 \times 64 \times 64$ node points $63 \times 63 \times 63$ control cell, which is a relatively coarse grid for this benchmark problem. Due to this fact, the non-linear advective terms were interpolated by the ninth-order WENO scheme. For the PP method itself, the Lax-Friedrichs scheme was employed with an explicit fourth-order Runge-Kutta time integration method.

Similarly to the two-dimensional benchmark problem, a mathematical task can be formulated in terms of boundary and initial conditions for the three-dimensional turbulent flow in a lid driven cubical cavity. For this test case, dimensionless quantities were used as well, thus the spatial coordinates were normalized by the characteristic length $L$ of the square cavity (see Fig. 18). The dimensional velocity components $u, v$ and $w$ were normalized by the average velocity $U_{b}$ of the moving lid on the top. No-slip boundary conditions were prescribed for the dimensionless velocity components $U, V$ and $W$ on the non-moving walls as $U_{\text {wall }}=V_{\text {wall }}=W_{\text {wall }}=0$. Dirichlet-type boundary conditions were imposed for the velocity components $U, V$ and $W$ on the moving lid as $U_{l i d}=1, V_{l i d}=0$, and $W_{l i d}=0$. It was essential to obtain a unique solution of the incompressible NavierStokes equations for this three-dimensional benchmark problem as well. Therefore the reference static pressure was set to be zero in the lower left corner of the front side of the cubical cavity. Thus Neumann-type boundary conditions were prescribed for the pressure on both moving and non-moving walls which means that the normal derivative of the pressure vanishes as $\partial P / \partial n=0$. The initial conditions for the velocity components $U$, $V$ and $W$ and the pressure values $P$ were equal to zero in each interior cell.

For this three-dimensional turbulent flow, the unsteady, incompressible Navier-Stokes equations were solved by using the Implicit Large Eddy Simulation (ILES) technique via a dual-time stepping procedure. The ILES method relying on the Godunov-procedure 


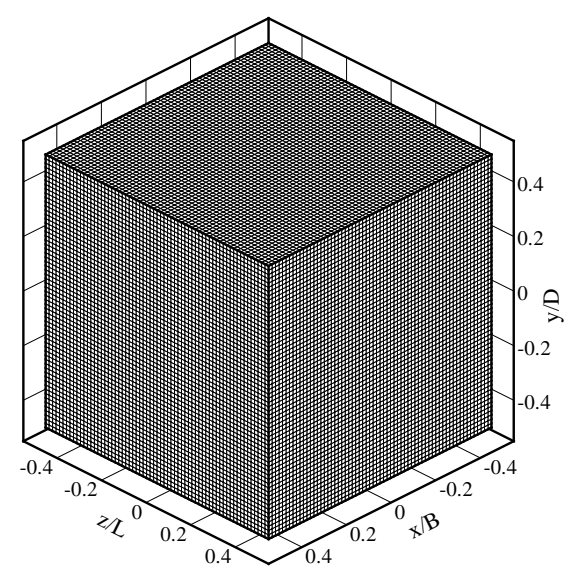

Figure 18: Equidistant numerical grid of the cubical cavity by using dimensionless coordinates, where $L=B=$ $D=150 \mathrm{~mm}$ with spanwise aspect ratio of $1: 1$.

has advantageous features for this problem, because a) the subgrid scale modelling is included implicitly in the numerical scheme employed for the non-linear convective terms, and $b$ ) the Godunov-type treatment of non-linear advective fluxes makes unnecessary any restrictions for the cell Reynolds number at high-speed flows $[2,8]$. By employing a dual-time stepping procedure for the AC and FSAC-PP methods, the pseudo-time stepping process has to be performed until the steady-state solution is achieved, then the process has to be advanced in real time. In this paper, the computational results were compared to the experimental data of [53, p. 212] along the horizontal and vertical centrelines of the mid-plane of the cubical cavity at $z / L=0$ (see Fig. 19).

For the turbulent flow problem in the lid driven cubical cavity, the third velocity component $W$ becomes significant due to the presence of strong velocity fluctuations, which have a high-frequency [53]. At the early stage of the flow field formation, a clockwiserotating primary large eddy appears in the upper right corner, which slowly flows downstream [46]. During this period of time, the counter-rotating downstream secondary eddy starts to develop from nearly the mid-point of the right side of the cubical cavity towards the lower right corner. In the finally developed flow field, the primary large eddy is located in the middle of the symmetry plane; two counter-rotating secondary eddies appear in the lower corners; and a third vortex formation appears slightly lower than the left upper corner [46,53] (see also the vortex structure in Fig. 17 [53, p. 209]).

The flow is strongly turbulent in the mid-plane of the cubical cavity at $R e=10000$, and the dimensionless mean velocity profiles, the root-mean-square (RMS) profiles, and the shear stress $\overline{U^{\prime} V^{\prime}}$ profiles are compared against the experimental data of [53], which can be seen in Fig. 19. The RMS profiles represent the normal stresses in the Reynolds stress tensor. By employing the ILES method, the elements of the Reynolds stress tensor are modelled implicitly, and these values can be recovered by spatial averaging. The relationship between the Reynolds Averaged Navier-Stokes (RANS) and the ILES method 
based turbulence modelling can be expressed by the Reynolds decomposition [2] as

$$
\mathbf{U}_{T}=\overline{\mathbf{U}}+\mathbf{U}^{\prime},
$$

where $\mathbf{U}_{T}$ is the instantaneous (turbulent) velocity field, $\overline{\mathbf{U}}$ is the spatial averaged mean velocity field, and $\mathbf{U}^{\prime}$ is the fluctuating velocity field. The instantaneous velocity field $\mathbf{U}_{I L E S}=\mathbf{U}_{T}$ was obtained as the result of the ILES method.

The spatial averaged mean velocity field may be computed by

$$
\overline{\mathbf{U}}=\frac{1}{N} \sum_{i, j, k}^{N}\left(\mathbf{U}_{I L E S}\right)_{i, j, k},
$$

where $N$ is the number of computational cells. Relying on the Reynolds decomposition (3.11), the fluctuating velocity field can be approximated by

$$
\mathbf{U}^{\prime}=\mathbf{U}_{I L E S}-\overline{\mathbf{U}},
$$

and by averaging the products of the fluctuating velocity components $U^{\prime}, V^{\prime}$ and $W^{\prime}$, the elements of the Reynolds stress tensor can be recovered. The mean velocity, normal and shear stress profiles were computed by employing the AC, PP and FSAC-PP methods in the framework of the ILES method [58].

All simulations have been performed on a Dual-Core E5300 $2.60 \mathrm{GHz}$ computer with the real-time step $\Delta t^{*}=0.01$ to measure computational times. The convergence criterion of the dual-time stepping procedure was $10^{-5}$ in each pseudo-time step for the AC and FSAC-PP methods. When the PP method itself was employed, 50 sub-iterations were performed by the S.O.R. method with $\omega=1.7$ relaxation factor for solving the pressurePoisson equation. The FSAC-PP method satisfies the continuity equation iteratively in the pseudo-time stepping procedure, thus 10 sub-iterations were used with the S.O.R. method for updating the pressure field. Note that when the FSAC-PP approach is employed, one sub-iteration is usually sufficient to solve the pressure-Poisson equation. For unsteady flows, it has been observed that the inclusion of the PP step in the AC formulation can speed up the satisfaction of the divergence-free (incompressibility) constraint in the perturbed continuity equation. Therefore the steady-state solution of the dualtime stepping procedure can be achieved faster with the FSAC-PP method than with the AC method in most cases. Furthermore, when the FSAC-PP approach is employed, it is not necessary to perform many sub-iterations for solving the pressure-Poisson equation compared to the PP method. For this benchmark problem, these reasons could justify that the computational time was decreased by employing the proposed FSAC-PP approach, compared to the AC and PP methods (see Table 14).

The numerical results showed that the AC, PP and FSAC-PP methods capture the mean velocity components better than the RMS normal and shear stress profiles when compared to the experimental data of [53]. The RMS stress profiles followed the tendency of the measured values with significant deviations between experiments and simulations, which were similar to the DNS results of Leriche et al. [55] at high Reynolds 

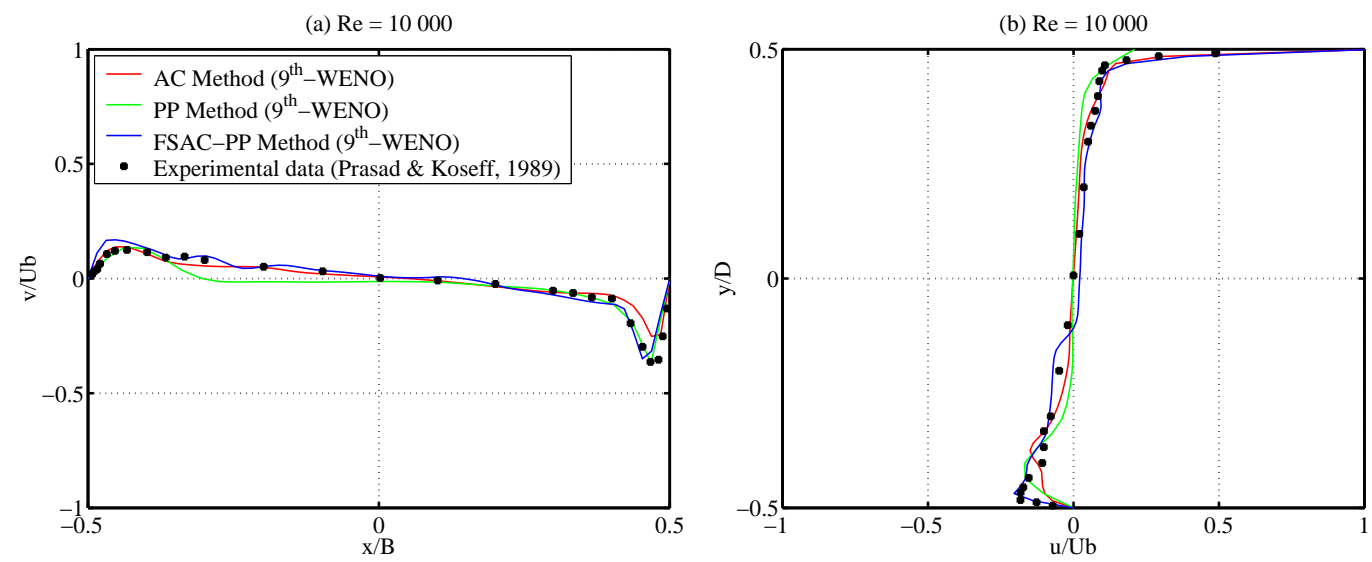

(c) $\operatorname{Re}=10000$
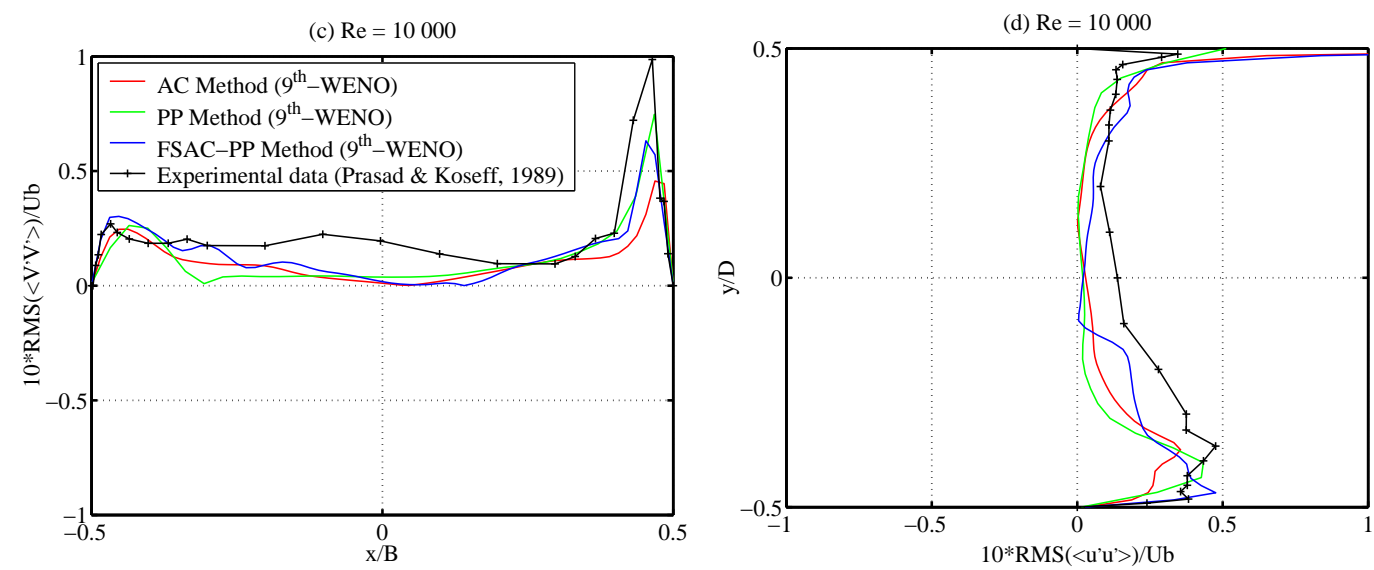

(e) $\operatorname{Re}=10000$
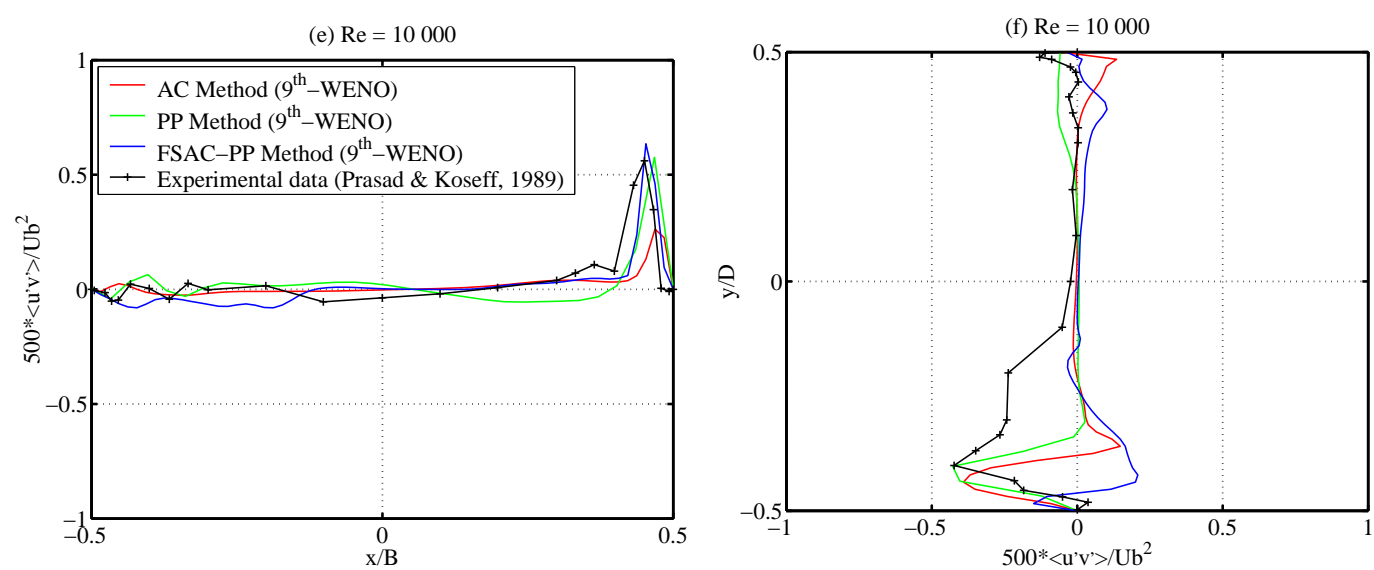

Figure 19: ILES results of the AC, PP, and FSAC-PP methods compared to the experimental data of Prasad et al. [53, p. 211] in the mid-plane $z / L=0$ at $R e=10000$; dimensionless mean velocity profiles along a) horizontal and b) vertical centre-lines; dimensionless normal stress profiles $10 \sqrt{\overline{V^{\prime 2}}} / U_{b}$ and $10 \sqrt{\overline{U^{\prime 2}}} / U_{b}$ along c) horizontal and d) vertical centre-lines; shear stress profiles $500 \sqrt{\overline{U^{\prime} V^{\prime}}} / U_{b}^{2}$ along e) horizontal and f) vertical centre-lines. 
Table 14: Computational times for an unsteady turbulent flow problem in a lid driven cubical cavity at $R e=10000$ on a Dual-Core E5300 $2.60 \mathrm{GHz}$ computer by employing $9^{r d}$-order WENO interpolation scheme [24-26] for the convective term.

\begin{tabular}{||c|c||}
\hline Methods & Computational Times of 41380 Time Iterations (Days) \\
\hline AC & 12 \\
\hline PP & 14.5 \\
\hline FSAC-PP & 7 \\
\hline
\end{tabular}

numbers. According to Leriche et al. [55], the differences in the fluctuating field between the simulation and the experiment are caused by the measurement errors approaching to the moving wall. Therefore the deviations observed in the current simulations may also be due to the same reason. Overall, the AC, PP, and FSAC-PP methods also lead to a good agreement with the experimental data for this higher Reynolds number flow.

\section{Conclusions}

In this paper, a unified fractional-step (FS), artificial compressibility (AC) and pressure projection (PP) formulation and numerical algorithm have been introduced in conjunction with a characteristics-based (CB) Godunov-type scheme for incompressible flows. The advantageous feature of the proposed FSAC-PP approach is the inclusion of the PP step in the AC formulation via pseudo-time splitting to decrease the magnitude of the pseudo-pressure term in the perturbed continuity equation, thus enforcing and accelerating the satisfaction of the continuity equation.

For simulating pressure-driven stationary laminar flows in a straight microfluidic channel, the AC method failed to give physically reasonable results at $\operatorname{Re}<0.1$, while both PP and FSAC-PP methods were capable of handling extremely low Reynolds number flows more accurately than the classical AC method. The results suggest that the proposed FSAC-PP method is valid for Reynolds numbers up to $10^{-4}$.

For two-dimensional stationary laminar flows in a lid driven square cavity, the results showed that the accuracy of the AC and FSAC-PP methods were similar to each other. For this benchmark problem, the FSAC-PP approach was the most efficient method compared to the AC and PP methods at $R e=100,400$, and 1000, respectively. Furthermore, the FSAC-PP approach was also capable of providing accurate and efficient solution by employing first-order (low-order) interpolation scheme for the convective flux terms compared to the use of higher-order schemes.

For a three-dimensional unsteady turbulent flow in a lid driven cubical cavity, the AC, PP and FSAC-PP methods captured the mean velocity profiles more accurately than the RMS normal and shear stress profiles. For this problem, the FSAC-PP approach performed better again than the AC and PP methods by decreasing the computational time.

The overall results suggest that the proposed FSAC-PP approach is more efficient than the AC and PP methods at low and moderate Reynolds numbers, and at a higher 
Reynolds number flow regime as well. The FSAC-PP method provides an advantageous numerical strategy both for stationary and unsteady flow problems relating to the presented benchmark problems. As a future work, it will be necessary to further investigate the FSAC-PP method for more complex flows regarding unsteady problems.

\section{Acknowledgments}

The authors would like to acknowledge Tommaso Oggian's assistance regarding the implementation of the pressure-Poisson solver.

\section{References}

[1] L. Könözsy, Multiphysics CFD Modelling of Incompressible Flows at Low and Moderate Reynolds Numbers, Ph.D. Thesis, Cranfield University, College of Aeronautics, Department of Engineering Physics, 2012.

[2] D. Drikakis, W. Rider, High-Resolution Methods for Incompressible and Low-Speed Flows, Springer-Verlag, Berlin, 2005.

[3] A. J. Chorin, A numerical method for solving incompressible viscous flow problems, J. Comput. Phys., 2 (1967), 12-26.

[4] R. Peyret, T. Taylor, Computational Methods for Fluid Flow, Springer-Verlag, Berlin, 1983.

[5] A. J. Chorin, Numerical solution of the Navier-Stokes equations, Math. Comp., 22 (1968), $745-762$.

[6] R. Temam, Sur l'approximation de la solution des equations de Navier-Stokes par la methode des pas fractionnaires (i), Arch. Rat. Mech. Anal., 32 (1969), 377-385.

[7] O. A. Ladyzhenskaya, Mathematical Problems in the Dynamics of a Viscous Incompressible Flow, Gordon and Breach, New York, 1963.

[8] J. B. Bell, P. Colella, H. M. Glaz, A second-order projection method for the incompressible Navier-Stokes equations, J. Comput. Phys., 85 (1989), 257-283.

[9] J. B. Bell, D. L. Marcus, A second-order projection method for variable-density flows, J. Comput. Phys., 101 (1992), 334-348.

[10] A. S. Almgren, J. B. Bell, P. Colella, L. H. Howell, M. L. Welcome, A conservative adaptive projection method for the variable density incompressible Navier-Stokes equations, J. Comput. Phys., 142 (1998), 1-46.

[11] R. B. Pember, L. H. Howell, J. B. Bell, P. Colella, W. Y. Crutchfield, W. A. Fiveland, J. P. Jessee, An adaptive projection method for unsteady low-Mach number combustion, Combust. Sci. Technol., 140 (1998), 123-168.

[12] J. Kim, P. Moin, Application of a fractional-step method to incompressible Navier-Stokes equations, J. Comput. Phys., 59 (1985), 308-323.

[13] J. B. Perot, An analysis of the fractional step method, J. Comput. Phys., 108 (1993), 51-58.

[14] J. B. Bell, P. Colella, J. A. Trangenstein, Higher order Godunov methods for general systems of hyperbolic conservation laws, J. Comput. Phys., 82 (1989), 362-397.

[15] P. Colella, Multidimensional upwind methods for hyperbolic conservation laws, J. Comput. Phys., 87 (1990), 171-200.

[16] A. Eberle, Characteristic flux averaging approach to the solution of Euler's equation, VKI Lecture Series, Computational Fluid Dynamics, 1987-04. 
[17] D. Drikakis, P. A. Govatsos, D. E. Papantonis, A characteristic-based method for incompressible flows, Int. J. Numer. Meth. Fl., 19 (1994), 667-685.

[18] D. Drikakis, O. P. Iliev, D. P. Vassileva, A nonlinear multigrid method for the threedimensional incompressible Navier-Stokes equations, J. Comput. Phys., 146 (1998), 301-321.

[19] D. Drikakis, A parallel multiblock characteristic-based method for three-dimensional incompressible flows, Advances in Engineering Software, 26 (1996), 111-119.

[20] E. Shapiro, D. Drikakis, Artificial compressibility, characteristics-based schemes for variable density, incompressible, multi-species flows. Part I. Derivation of different formulations and constant density limit, J. Comput. Phys., 210 (2005), 584-607.

[21] E. Shapiro, D. Drikakis, Artificial compressibility, characteristics-based schemes for variabledensity, incompressible, multispecies flows: Part II. Multigrid implementation and numerical tests, J. Comput. Phys., 210 (2005), 608-631.

[22] E. Shapiro, D. Drikakis, J. Gargiuli, P. Vadgama, Interface capturing in dual-flow microfluidics, J. Comput. Theor. Nanosci., 4 (4) (2007), 802-806.

[23] K. Zamzamian, S. E. Razavi, Multidimensional upwinding for incompressible flows based on characteristics, J. Comput. Phys., 227 (2008), 8699-8713.

[24] G.-S. Jiang, C.-W. Shu, Efficient implementation of weighted ENO schemes, J. Comput. Phys., 126 (1996), 202-228.

[25] C.-W. Shu, Essentially non-oscillatory and weighted essentially non-oscillatory schemes for hyperbolic conservation laws, NASA/CR-97-206253, ICASE Report No. 97-65, 1997.

[26] D. S. Balsara, C.-W. Shu, Monotonicity preserving weighted essentially non-oscillatory schemes with increasingly high order of accuracy, J. Comput. Phys., 160 (2000), 405-452.

[27] H. S. Tang, F. Sotiropoulos, Fractional step artificial compressibility schemes for the unsteady incompressible Navier-Stokes equations, Comput. Fluids, 36 (2007), 974-986.

[28] O. C. Zienkiewicz, R. Codina, A general algorithm for compressible and incompressible flow - Part I. The split, characteristic-based scheme, Int. J. Numer. Meth. Fl., 20 (1995), 869-885.

[29] O. C. Zienkiewicz, K. Morgan, B. V. K. S. Sai, R. Codina, M. Vasquez, A general algorithm for compressible and incompressible flow - Part II. Tests on the explicit form, Int. J. Numer. Meth. Fl., 20 (1995), 887-913.

[30] O. C. Zienkiewicz, B. V. K. S. Sai, K. Morgan, R. Codina, Split, characteristic based semiimplicit algorithm for laminar/turbulent incompressible flows, Int. J. Numer. Meth. Fl., 23 (1996), 787-809.

[31] O. C. Zienkiewicz, P. Nithiarasu, R. Codina, M. Vázquez, P. Ortiz, The characteristic-basedsplit procedure: An efficient and accurate algorithm for fluid problems, Int. J. Numer. Meth. Fl., 31 (1999), 359-392.

[32] R. Codina, M. Vázquez, O. C. Zienkiewicz, A general algorithm for compressible and incompressible flows. Part III: The semi-implicit form, Int. J. Numer. Meth. Fl., 27 (1998), 13-32.

[33] P. Nithiarasu, On boundary conditions of the characteristic based split (CBS) algorithm for fluid dynamics, Int. J. Numer. Meth. Engng., 54 (2002), 523-536.

[34] P. Nithiarasu, An efficient artificial compressibility (AC) scheme based on the characteristic based split (CBS) method for incompressible flows, Int. J. Numer. Meth. Engng., 56 (2003), 1815-1845.

[35] P. Nithiarasu, J. S. Mathur, N. P. Weatherill, K. Morgan, Three-dimensional incompressible flow calculations using the characteristic based split (CBS) scheme, Int. J. Numer. Meth. Fl., 44 (2004), 1207-1229.

[36] P. Nithiarasu, R. Codina, O. C. Zienkiewicz, The characteristic-based split (CBS) scheme - a unified approach to fluid dynamics, Int. J. Numer. Meth. Engng., 66 (2006), 1514-1546. 
[37] P. Nithiarasu, O. C. Zienkiewicz, Analysis of an explicit and matrix free fractional step method for incompressible flows, Comput. Methods Appl. Mech. Engrg., 195 (2006), 55375551.

[38] P. Nithiarasu, R. L. T. Bevan, K. Murali, An artificial compressibility based fractional step method for solving time dependent incompressible flow equations. temporal accuracy and similarity with a monolithic method, Comput. Mech., 51 (2013), 255-260.

[39] R. Courant, D. Hilbert, Methods of Mathematical Physics, John Wiley and Sons Inc., New York, 1991.

[40] C.-W. Shu, S. Osher, Efficient implementation of essentially non-oscillatory shock-capturing schemes, J. Comput. Phys., 77 (1988), 439-471.

[41] M. F. Tome, S. McKee, GENSMAC - A computational marker and cell method for free surface flows in general domains, J. Comput. Phys., 110 (1994), 171-186.

[42] P. Roache, Computational Fluid Dynamics, Albuquerque: Hermosa, 1976.

[43] G. Karniadakis, A. Beskok, N. Aluru, Microflows and Nanoflows, Springer, New York, 2005.

[44] G. E. Karniadakis, S. Sherwin, Spectral/hp Element Methods for Computational Fluid Dynamics (2nd ed), Oxford University Press, 2005.

[45] G. E. Karniadakis, M. Israeli, S. A. Orszag, High-order splitting methods for the incompressible Navier-Stokes equations, J. Comput. Phys., 97 (1991), 414-443.

[46] M. Griebel, T. Dornseifer, T. Neunhoeffer, Numerical Simulation in Fluid Dynamics, Society for Industrial and Applied Mathematics (SIAM), 1998.

[47] J.-L. Guermond, C. Migeon, G. Pineau, L. Quartapelle, Start-up flows in a three-dimensional rectangular driven cavity of aspect ratio 1:1:2 at $\operatorname{Re}=1000$, J. Fluid Mech., 450 (2002), 169199.

[48] S. Kotake, K. Hijikata, T. Fusegi, Numerical Simulations of Heat Transfer and Fluid Flow on a Personal Computer, Transport Processes in Engineering, Elsevier, London, 19933.

[49] X. Y. Chen, K. C. Toh, J. C. Chai, C. Yang, Developing pressure-driven liquid flow in microchannels under the electrokinetic effect, Int. J. Eng. Sci. 42 (2004), 609-622.

[50] S. Chakraborty, Augmentation of peristaltic microflows through electroosmotic mechanisms, J. Phys. D: Appl. Phys., 39 (2006), 5356-5363.

[51] S. Chakraborty, Electroosmotically driven capillary transport of typical non-Newtonian biofluids in rectangular microchannels, Anal. Chim. Acta, 605 (2007), 175-184.

[52] U. Ghia, K. N. Ghia, C. T. Shin, High-Re solutions for incompressible flow using the NavierStokes equations and a multigrid method, J. Comput. Phys., 48 (1982), 387-411.

[53] A. K. Prasad, J. R. Koseff, Reynolds number and end-wall effects on a lid-driven cavity flow, Phys. Fluids A, 1 (2) (1989), 208-218.

[54] W. Y. Soh, J. W. Goodrich, Unsteady solution of incompressible Navier-Stokes equations, J. Comput. Phys., 79 (1988), 113-134.

[55] E. Leriche, S. Gavrilakis, M. O. Deville, A spectral direct simulation method for a 3D inhomogeneous domain, 24th Workshop Proceedings, Speedup Journal, 12 (2) (1998), 17-21.

[56] E. Leriche, Direct numerical simulation in a lid-driven cubical cavity at high Reynolds number by a Chebyshev spectral method, SIAM J. Sci. Comput., 27 (1-3) (2006), 335-345.

[57] L. H. Olesen, Computational Fluid Dynamics in Microfluidic Systems, M.Sc. Thesis, Mikroelektronik Centret, Technical University of Denmark, 2003.

[58] F. F. Grinstein, L. G. Margolin, W. J. Rider, Implicit Large Eddy Simulation: Computing Turbulent Fluid Dynamics, Cambridge University Press, 2007. 
2014-08-28

\section{A unified fractional-step, artificial} compressibility and pressure-projection formulation for solving the incompressible Navier-Stokes equations

Könözsy, László Z.

Global Science Press

Könözsy, L., Drikakis, D. A Unified fractional-step, artificial compressibility and pressure-projection formulation for solving the incompressible Navier-stokes equations (2014) Communications in Computational Physics, 16 (5), pp. 1135-1180.

https://doi.org/10.4208/cicp.240713.080514a Downloaded from Cranfield Library Services E-Repository 